\title{
Expression of mitochondrial membrane-linked SAB determines severity of sex-dependent acute liver injury
}

\author{
Sanda Win, ${ }^{1}$ Robert W.M. Min, ${ }^{1}$ Christopher Q. Chen, ${ }^{1}$ Jun Zhang, ${ }^{2}$ Yibu Chen, ${ }^{3}$ Meng Li, ${ }^{3}$ Ayako Suzuki, ${ }^{4}$ Manal F. Abdelmalek, ${ }^{4}$ \\ Ying Wang, ${ }^{4}$ Mariam Aghajan, ${ }^{5}$ Filbert W.M. Aung, ${ }^{1}$ Anna Mae Diehl, ${ }^{4}$ Roger J. Davis, ${ }^{6}$ Tin A. Than, ${ }^{1}$ and Neil Kaplowitz ${ }^{1}$ \\ 'USC Research Center for Liver Disease, Keck School of Medicine, University of Southern California (USC), Los Angeles, California, USA. Department of Gastroenterology, Renmin Hospital of Wuhan University, \\ Wuhan, China. ${ }^{3}$ USC Libraries Bioinformatics Service, Norris Medical Library, USC, Los Angeles, California, USA. ${ }^{4}$ Division of Gastroenterology, Department of Medicine, Duke University, Durham, North Carolina, \\ USA. ${ }^{5}$ Ionis Pharmaceuticals, Carlsbad, California, USA. ${ }^{6}$ Howard Hughes Medical Institute and Program in Molecular Medicine, University of Massachusetts Medical School, Worcester, Massachusetts, USA.
}

\begin{abstract}
SH3 domain-binding protein that preferentially associates with Btk (SAB) is an outer-membrane docking protein for JNKmediated impairment of mitochondrial function. Deletion of Sab in hepatocytes inhibits sustained JNK activation and cell death. The current study demonstrates that an increase in SAB expression enhanced the severity of acetaminophen-induced (APAP-induced) liver injury. Female mice were resistant to liver injury and exhibited markedly decreased hepatic SAB protein expression compared with male mice. The mechanism of SAB repression involved a pathway from ER $\alpha$ to $p 53$ expression that induced miR34a-5p. miR34a-5p targeted the Sab mRNA coding region, thereby repressing SAB expression. Fulvestrant or $p 53$ knockdown decreased miR34a-5p and increased SAB expression in female mice, leading to increased injury from APAP and TNF/galactosamine. In contrast, an ER $\alpha$ agonist increased p53 and miR34a-5p, which decreased SAB expression and hepatotoxicity in male mice. Hepatocyte-specific deletion of miR34a also increased the severity of liver injury in female mice, which was prevented by GaINAc-ASO knockdown of Sab. Similar to mice, premenopausal women expressed elevated levels of hepatic $p 53$ and low levels of SAB, whereas age-matched men expressed low levels of $p 53$ and high levels of SAB, but there was no difference in SAB expression between the sexes in the postmenopausal stage. In conclusion, SAB expression levels determined the severity of JNK-dependent liver injury. Female mice expressed low levels of hepatic SAB protein because of the ER $\alpha / \mathrm{p} 53 / \mathrm{miR} 34 a$ pathway, which repressed SAB expression and accounted for the resistance to liver injury seen in these females.
\end{abstract}

\section{Introduction}

JNK signaling is important in liver injury scenarios (1-8) and appears to involve the interaction of activated JNK and an outer mitochondrial membrane target, $\mathrm{SH} 3$ domain-binding protein 5 (SAB, also known as SH3BP5) (9). This interaction has been extensively studied in the acetaminophen (APAP) hepatotoxicity model $(10,11)$, as well as in other models (12-14). A marked sex difference in susceptibility to toxicity has been well documented in APAP-treated mice (female resistance), but the mechanism for this sex difference is not fully understood (15-17). We hypothesized that this sex difference may not be unique to APAP and may be indicative of a sex difference in the contribution of JNK-SAB interaction.

The mechanism of APAP hepatotoxicity involves the conversion of a small proportion of APAP to a toxic intermediate, NAPQI, which initially depletes glutathione (GSH), leaving unprotected protein thiols that undergo covalent binding, especially in mitochondria. This impairs mitochondrial function to an extent sufficient to release ROS, which activate the MAPK cascade leading

Authorship note: SW and RWMM are co-first authors.

Conflict of interest: The authors have declared that no conflict of interest exists. Copyright: (c) 2019, American Society for Clinical Investigation.

Submitted: February 25, 2019; Accepted: August 29, 2019; Published: October 28, 2019.

Reference information: / Clin Invest. 2019;129(12):5278-5293.

https://doi.org/10.1172/JCl128289. to JNK activation (18). JNK then binds to and phosphorylates SAB on the outer membrane. $\mathrm{SAB}$ then releases a protein phosphatase (PTPN6) bound to the intermembrane face of SAB, leading to its interaction with phosphorylated SRC (p-SRC) (active), facilitated by the inner-membrane platform protein DOK4. p-SRC is then inactivated, leading to inhibition of mitochondrial electron transport (which requires activated SRC) and increased ROS release, sustaining JNK activation and amplifying ROS exposure in mitochondria $(11,12)$ and ultimately leading to necrosis through the mitochondrial permeability transition (MPT) pore $(19,20)$. Pivotal in this context is the maintenance of sustained JNK activation. This pathway, referred to as the JNK/SAB/ROS activation loop, is also important in the mechanism of TNF-induced apoptosis of hepatocytes sensitized by treatment with D-galactosamine (GalN) in vivo or actinomycin D (ActD) in vitro $(10,11)$. In this circumstance, sustained JNK activation modulates the BCL family of gatekeepers of outer mitochondrial membrane permeability by activating BID, BAX, BIM, and PUMA and inactivating BCL- $\mathrm{X}_{\mathrm{L}}$ and MCL-1 (21-26). We previously showed that hepatocyte-specific SAB deletion in male mice prevented sustained JNK activation and liver injury in the APAP- and GalN/TNF-induced liver injury models. Since an earlier study showed no sex difference in total hepatic JNK1 and JNK2 and decreased sustained JNK activation in female mice in an APAP injury model (17), we hypothesized that sex-dependent regulation of SAB expression may account for the 
A

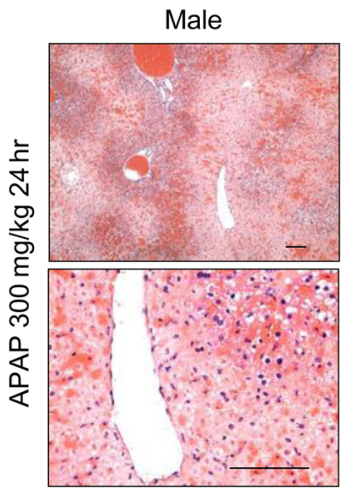

ALT $14000 \pm 2000 \mathrm{U} / \mathrm{L}$

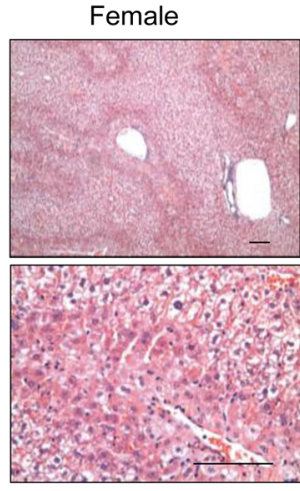

$2000 \pm 1000 \mathrm{U} / L^{*}$

C
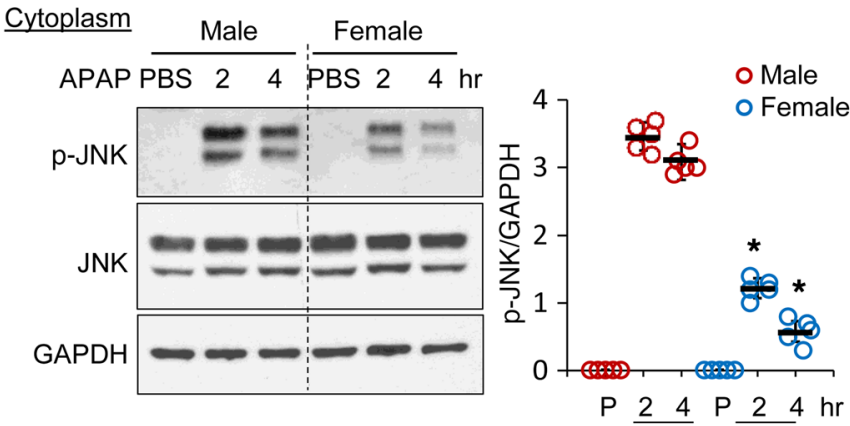

Mitochondria
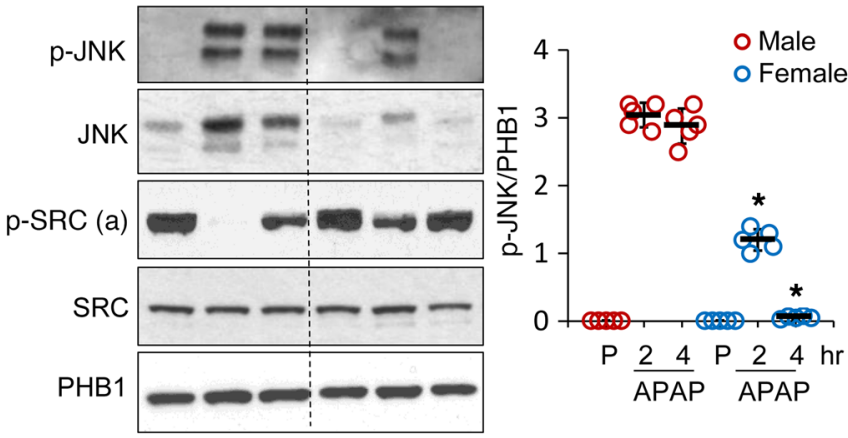

B

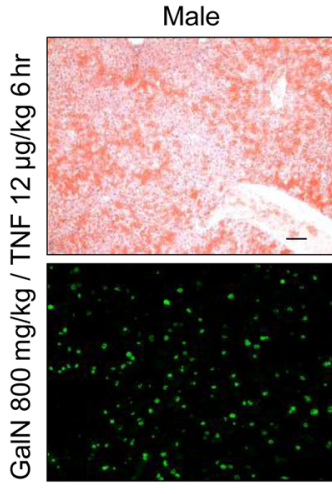

$\mathrm{ALT} 9000 \pm 500 \mathrm{U} / \mathrm{L}$

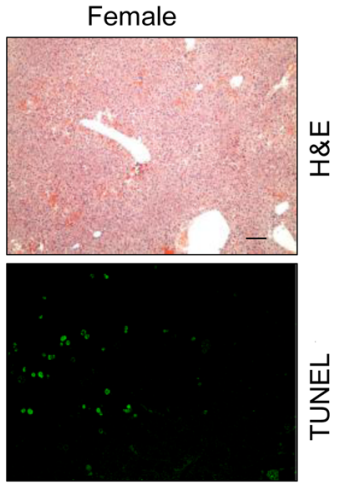

$200 \pm 50 \mathrm{U} / \mathrm{L}^{*}$

D

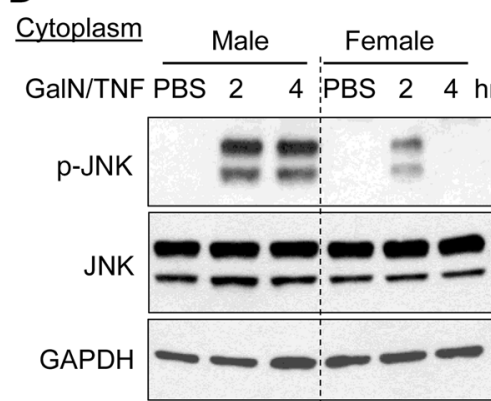

Mitochondria

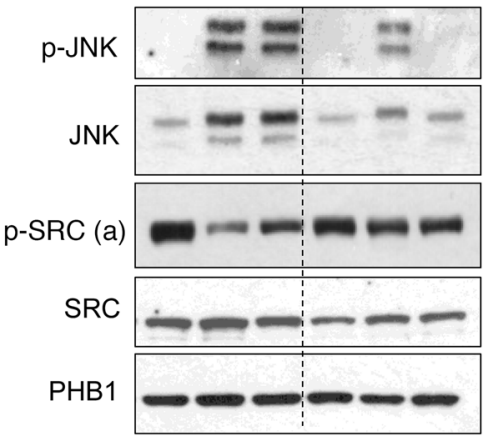

- Male - Female
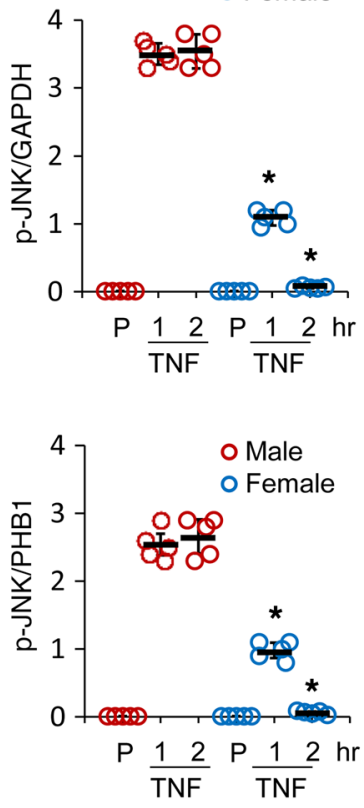

Figure 1. Sex difference in APAP-induced necrotic or GaIN/TNF-induced apoptotic liver injury. (A and B) Ten- to twelve-week-old C57BL/6N WT male and female littermates were fasted overnight and treated. Serum ALT levels were measured and H\&E staining was performed 24 hours after APAP (300 mg/kg) treatment. ALT levels were measured and H\&E, and TUNEL staining was performed 6 hours after treatment with GalN $(800 \mathrm{mg} / \mathrm{kg})$ and TNF (12 $\mu \mathrm{g} / \mathrm{kg})$. Scale bars: $100 \mu \mathrm{m} . n=5$ mice/group. ${ }^{*} P<0.05$ versus male mice, by unpaired, 2 -tailed Student's $t$ test. Data are presented as the mean \pm SD. (C) Liver cytoplasm and mitochondria from male and female littermates were isolated by differential centrifugation after PBS treatment or 2 or 4 hours after APAP treatment. (D) Liver cytoplasm and mitochondria from male and female littermates were isolated by differential centrifugation after PBS treatment or 1 or 2 hours after GaIN/PBS or GaIN/TNF treatment. ${ }^{*} P<0.05$ versus male, by 1-way ANOVA with Bonferroni's correction. Data are presented as the mean \pm SEM. Protein extract ( $30 \mu \mathrm{g}$ ) from cytoplasm or mitochondria was loaded for immunoblot analysis using anti-p-JNK, anti-JNK, anti-p-SRC (active), and anti-c-SRC. GAPDH (cytoplasm) and PHB1 (mitochondria) were used as loading controls. Representative immunoblots are from 3 separate experiments. $n=5$ mice/group. P, PBS.

sex difference in susceptibility to acute liver injury. More important, if this were to be the case, it would provide a unique opportunity to examine the regulation of SAB expression for the first time and to determine the importance of SAB expression levels in the susceptibility to and severity of acute liver injury.

\section{Results}

Resistance of female mice to acute liver injury is associated with low $S A B$ expression levels. Since liver injury induced by APAP is known to be more severe in the $\mathrm{N}$ substrain than in the J substrain of
C56BL/6 mice $(27,28)$, our first objective was to confirm that the previously reported resistance to APAP toxicity in the female C57BL/6J strain also extends to the C57BL/6N strain. The N substrain females had markedly decreased histologic necrosis and alanine aminotransferase (ALT) levels compared with littermate males (Figure 1A), accompanied by decreased sustained p-JNK activation and mitochondrial translocation but no sex difference in total JNK expression (Figure 1C). Furthermore, we confirmed prior reports that there is no sex difference in basal hepatic GSH levels or GCLC expression (Supplemental Figure 1, A and B). Fur- 
A

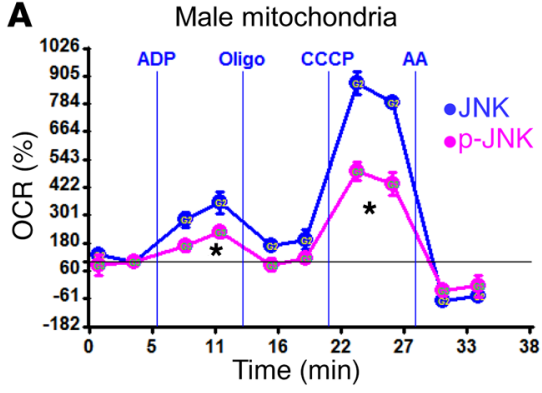

Female mitochondria

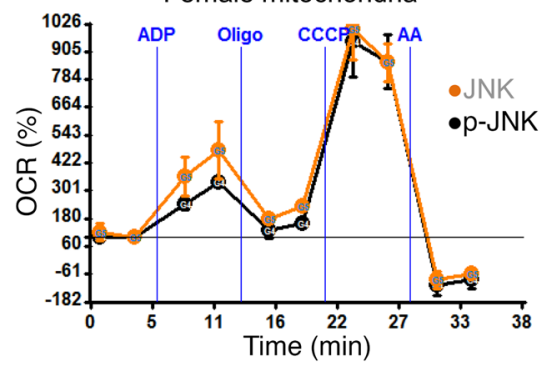

B

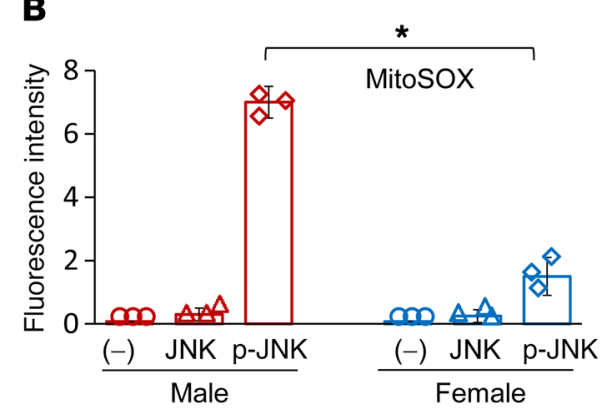

C

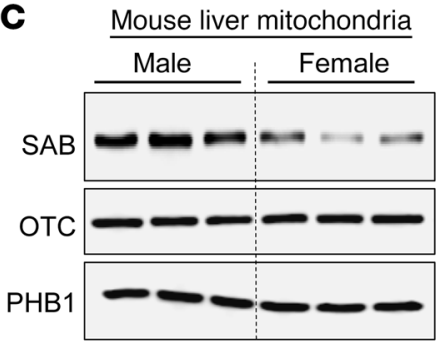

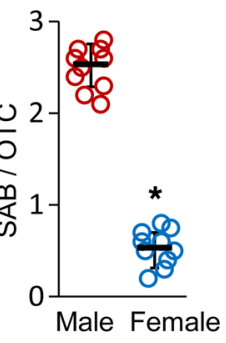

D

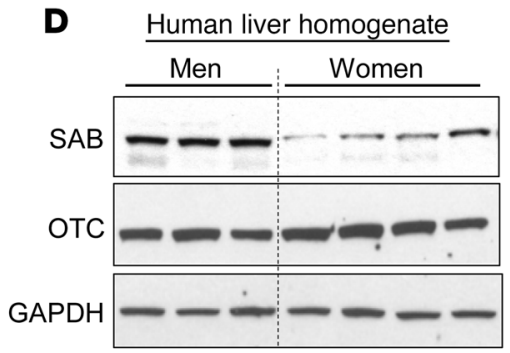

E

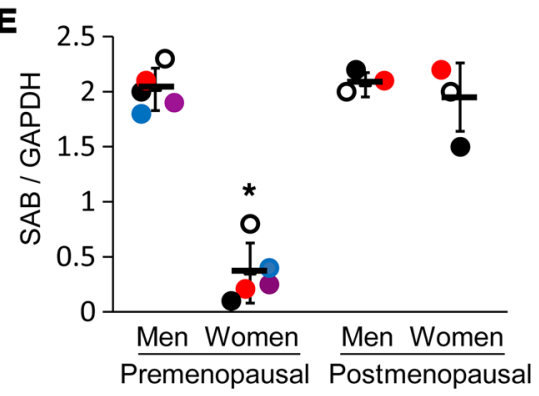

Figure 2. Sex difference in p-JNK inhibition of mitochondrial respiration and SAB expression. (A) Isolated mitochondria from male or female littermates were incubated with ATP plus recombinant JNK (isoforms 1 + 2) or p-JNK (isoforms $1+2$ ) and then ADP, oligomycin (Oligo), CCCP, or antimycin A (AA) were injected sequentially, and the OCR was measured with a Seahorse XF analyzer to determine state 3 and maximal respiration. Traces are representative of 5 separate experiments. ${ }^{*} P<0.05$ versus JNK, by 1-way ANOVA with Bonferroni's correction. Data are presented as the mean \pm SD of multiple wells for each treatment. (B) Mitochondria were isolated as for the mitochondrial respiration assay. Mitochondria were preincubated with MitoSOX and then treated with JNK or p-JNK with ATP, and fluorescence intensity was measured to determine superoxide production. $n=3$ mice/group. ${ }^{*} P<0.05$ versus males, by paired, 2 -tailed Student's $t$ test. (C) Mitochondria from WT male and female littermates were immunoblotted with anti-SAB, anti-OTC, or anti-PHB1. Representative immunoblot from 3 separate experiments. $n=10$ mice/group. ${ }^{*} P<0.05$ versus male mice, by paired, 2 -tailed Student's $t$ test. (D) Immunoblot analysis of SAB expression in representative human liver homogenates from premenopausal women and matched men. (E) Age- and BMI-matched men and women were divided into the premenopausal group or the postmenopausal group according to serum estradiol (E2) and FSH levels and menstrual and gynecological history (see Supplemental Table 1). In each group, the male and female pairs are indicated by the same color symbol in the figure. All liver biopsy samples were histologically classified as mild, nonspecific abnormalities. SAB and GAPDH levels were determined by Western blotting and densitometry. $n=5$ pairs in the premenopausal group and $n=3$ pairs in the postmenopausal group. ${ }^{*} P<0.05$ versus men, by paired, 2 -tailed Student's $t$ test. Data are presented as the mean \pm SEM.

thermore, the reported and confirmed greater recovery of GSH in females at 4 hours (Supplemental Figure 1A) corresponded with decreased p-JNK and injury (Figure 1). GCLC protein levels did not increase or decrease in female mice but rapidly declined in male mice before significant necrosis occurred (Supplemental Figure 1C), as we reported previously in male mice (29). Furthermore, we observed no sex difference in the rate of GSH recovery following depletion by nontoxic phorone (Supplemental Figure 1F), indicating no intrinsic sex difference in GSH synthesis. In addition, as reported in the B6J mouse substrain, we observed no sex difference in APAP metabolism to NAPQI, as reflected in APAP protein adduct formation in the B6N substrain (Supplemental Figure 1G). Nevertheless, because of the possibility that there may be inherent sex differences in other aspects of drug metabolism and antioxidant defense, we examined the GalN/TNF model of innate immune-mediated apoptotic cell death, which is independent of drug metabolism and GSH. Again, we observed a marked protection in female mice with respect to liver injury and JNK activation (Figure 1, B and D). In addition APAP induced greater dose-related $(0,100,200$, and $300 \mathrm{mg} / \mathrm{kg})$ liver injury in male mice compared with female mice as reflected in serum ALT elevation (Supplemental Figure 1D), and we noted a direct correlation between ALT and p-JNK 4 hours after APAP $(300 \mathrm{mg} / \mathrm{kg})$ treatment (Supplemen- tal Figure 1F). We found that cell-autonomous killing of primary mouse hepatocytes (PMHs) by APAP and ActD/TNF was significantly greater in males than in females (Supplemental Figure 1, $\mathrm{H}$ and I). In addition, using PMHs, we examined 2 other models in which toxicity is JNK and SAB dependent, i.e., tunicamycininduced (severe endoplasmic reticulum stress) (Supplemental Figure 1K) and palmitic acid-induced (Supplemental Figure 1J) apoptosis, and we observed a significant decrease in PMH death among female mice compared with male mice. In addition, we found no sex difference in basal expression of GRP78, PERK, ATF6, IRE1 $\alpha$, ATF2, c-JUN, MCL-1, or BCL- $\mathrm{X}_{\mathrm{L}}$ (Supplemental Figure 2).

The results for both APAP and GalN/TNF models of acute injury showed marked suppression of both p-JNK translocation to mitochondria and sustained p-JNK activation in the cytoplasm in female mice. The cause of sustained of p-JNK is release of ROS from mitochondria due to the intramitochondrial signaling pathway that leads to the dephosphorylation of p-SRC in the mitochondrial intermembrane space (11). Therefore, we examined the sex difference in terms of mitochondrial SRC inactivation, which was also blunted in both injury models in females (Figure 1, C and D). This suggested that mitochondria in female mice are resistant to the effect of p-JNK. To support this idea, we measured the direct inhibitory effect of p-JNK on normal mitochondrial respiration. As we report- 
A

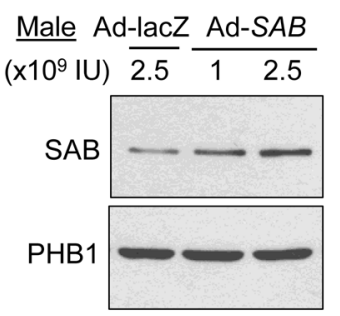

B

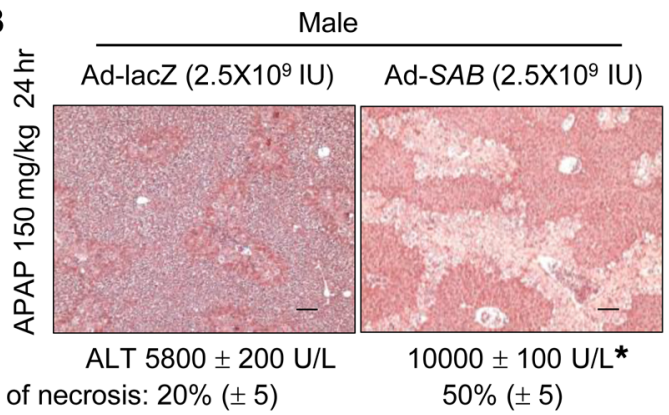

C
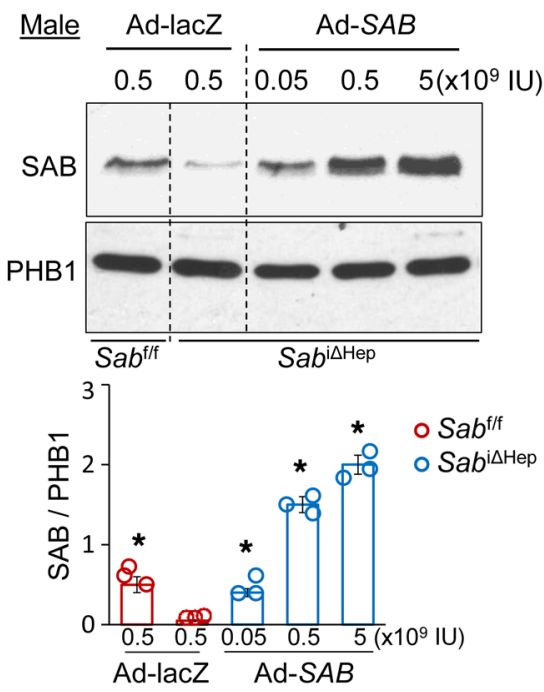

D

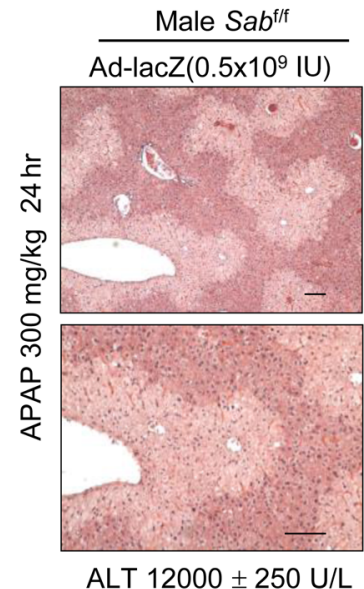

Area of necrosis: $45 \%( \pm 5)$

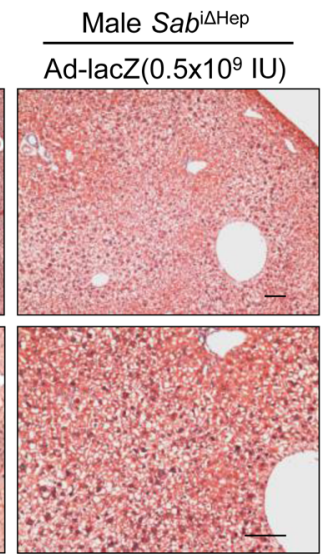

$90 \pm 50 \mathrm{U} / \mathrm{L}^{\star}$

$0 \%$

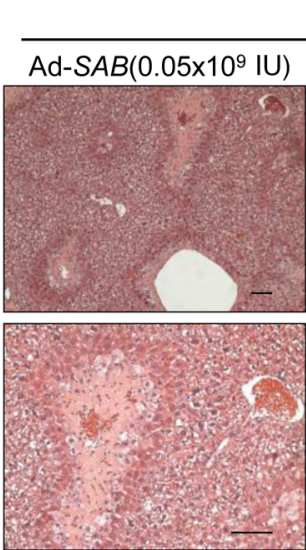

$7300 \pm 320 \mathrm{U} / \mathrm{L}^{* *}$ $20 \%( \pm 3)$

Male $S a b^{i \Delta \text { Hep }}$

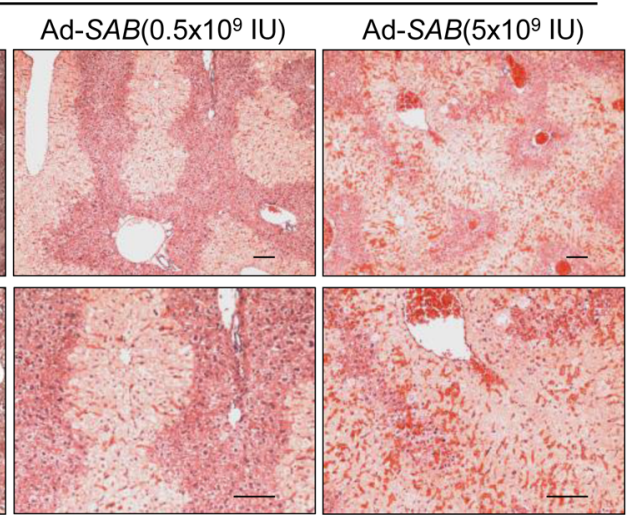

$12500 \pm 790 \mathrm{U} / \mathrm{L}^{\star \star}$ $55 \%( \pm 5)$

$14000 \pm 550 \mathrm{U} / \mathrm{L}^{\star *}$

$90 \%( \pm 5)$
E

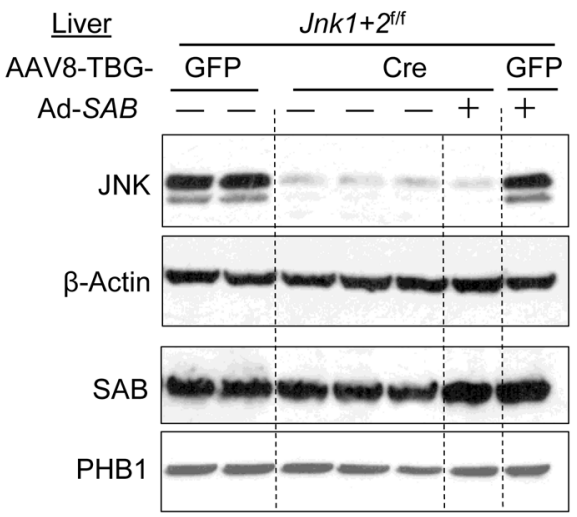

$\mathbf{F}$

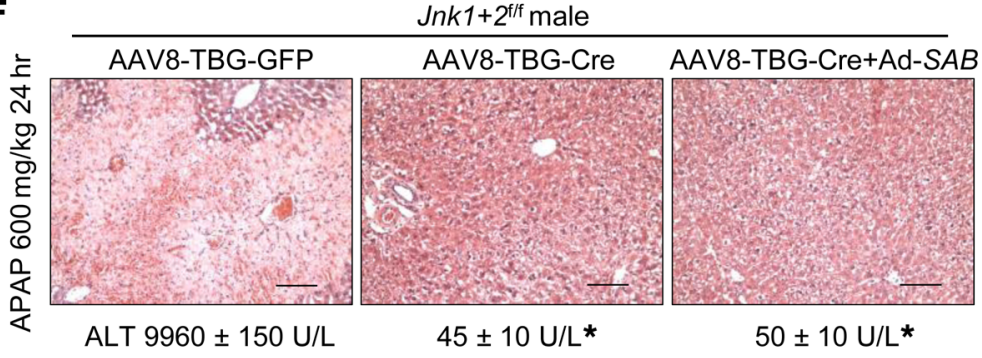

Figure 3. SAB expression in male mice determines the susceptibility to APAP-induced liver injury in a JNK-dependent fashion. WT C57BL/6N male mice received Ad-lacZ or Ad-SAB. (A) Fourteen days later, SAB expression was analyzed by immunoblotting, and (B) APAP (150 mg/kg) was given i.p., and H\&E staining was performed and serum ALT levels were determined after 24 hours. Original magnification, $\times 10$. Scale bars: $100 \mu \mathrm{m}$. $n=3 /$ group. ALT: ${ }^{*} P<0.05$ versus Ad-lacZ-injected Sab ${ }^{\text {is Hep }}$ mice, by unpaired, 2-tailed Student's $t$ test. Data are presented as the mean \pm SD. (C) Sab ${ }^{f / f l}$ or Sabistep mice received 0.05 $5 \times 10^{9}$ to approximately $5 \times 10^{9} \mathrm{IU}$ Ad-lacZ or Ad-SAB. Fourteen days later, SAB expression was determined by immunoblotting. Immunoblot is representative of 3 separate experiments. $n=3$ /group. ${ }^{*} P<0.05$ versus Sab ${ }^{\text {iAHep }}+$ Ad-lacZ, by 1-way ANOVA with Bonferroni's correction. Data are presented as the mean \pm SEM. (D) Mice received APAP ( $300 \mathrm{mg} / \mathrm{kg}$, i.p.), and 24 hours later, liver sections were stained with $\mathrm{H} \& \mathrm{E}$, and the necrotic area (percentage) was measured. Serum ALT ( \pm SEM) (U/L) was measured. Scale bars: $100 \mu \mathrm{m} . n=3$ mice/group. ALT: ${ }^{*} P<0.05$, versus Ad-lacZ-injected Sab ${ }^{f / f l}$ mice; ${ }^{* *} P<$ 0.05 , versus Ad-lacZ-injected Sab ${ }^{i \mathrm{HHep}}$ mice, by unpaired, 2-tailed Student's $t$ test. Data are presented as the mean $\pm \mathrm{SD}$. (E and $\left.\mathbf{F}\right) / n k 1 / 2^{f / f f l}$ mice received AAV8-TBG-GFP ( $n=3)$, AAV8-TBG-Cre $(n=5)$, or AAV8-TBG-Cre plus Ad-SAB $(n=3)$. Fourteen days later, JNK and SAB expression levels were determined by immunoblotting, or mice were treated with APAP to analyze liver injury and serum ALT levels. Scale bars: $100 \mu \mathrm{m}$. ALT: ${ }^{*} P<0.05$, versus AAV8-TBG-GFP, by unpaired, 2-tailed Student's $t$ test. Data are presented as the mean \pm SD. 
A

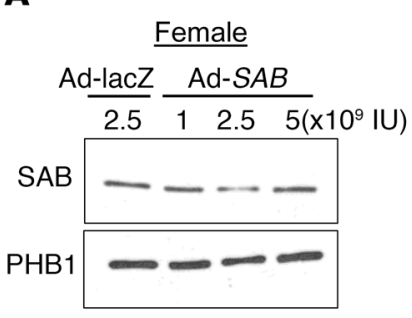

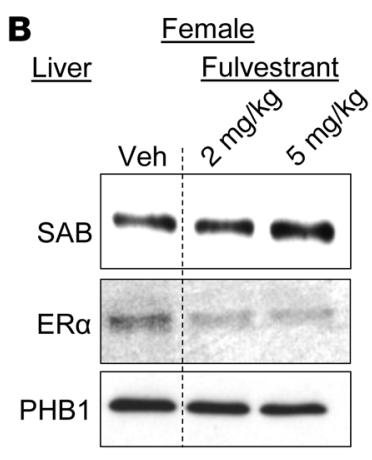

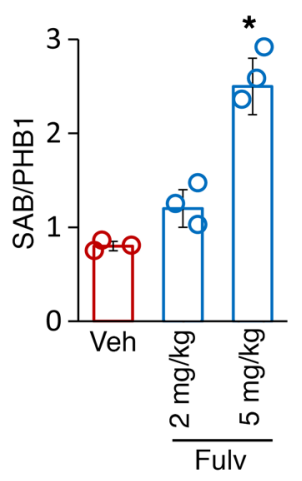

E

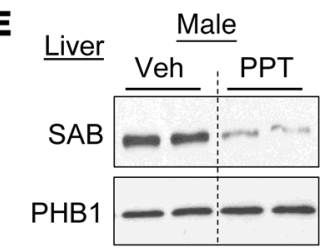

C

Liver Fulvestrant $(2 \mathrm{mg} / \mathrm{kg})$

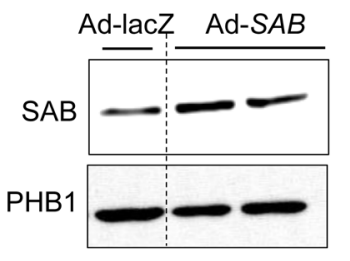

D

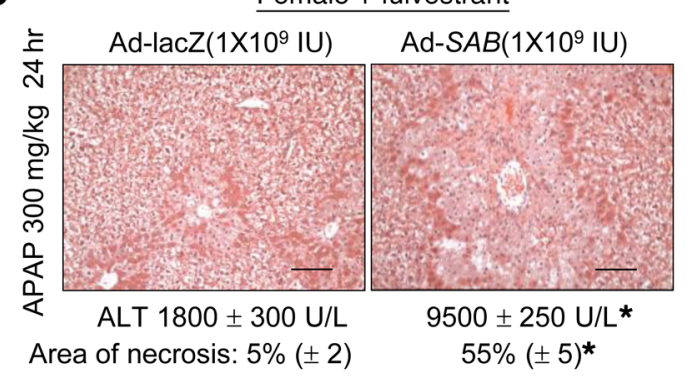

$\mathbf{F}$

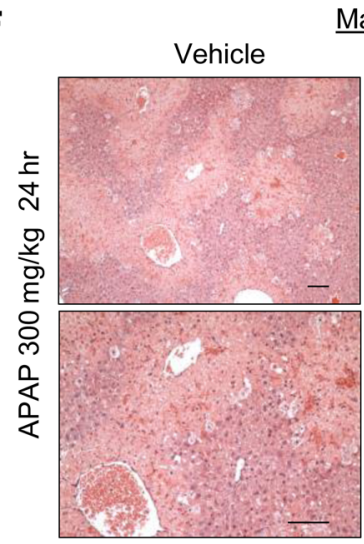

ALT $13800 \pm 420 \mathrm{U} / \mathrm{L}$ Area of necrosis: $50 \%( \pm 5)$

Male

PPT $5 \mathrm{mg} / \mathrm{kg}$

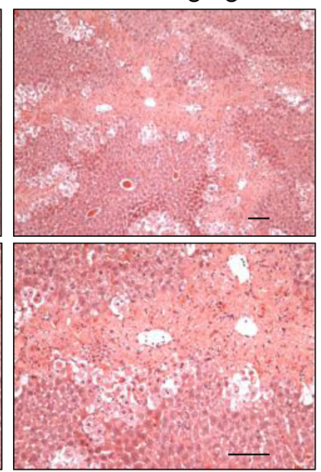

$2000 \pm 600 \mathrm{U} / \mathrm{L}^{\star}$ $15 \%( \pm 5)^{*}$

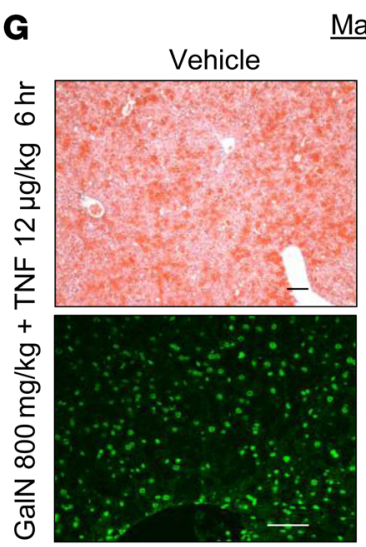

ALT $9300 \pm 120 \mathrm{U} / \mathrm{L}$
Male

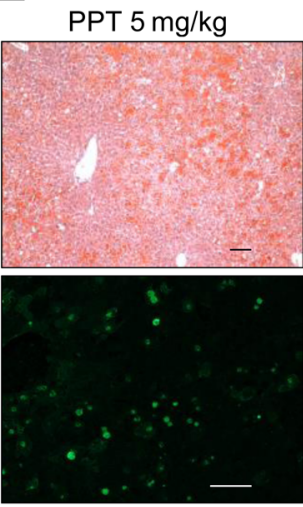

$1000 \pm 170 \mathrm{U} / \mathrm{L}^{*}$

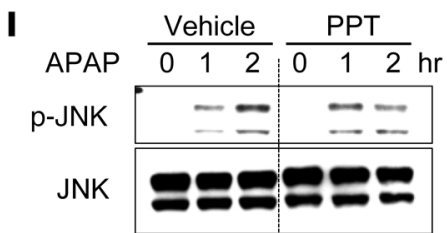

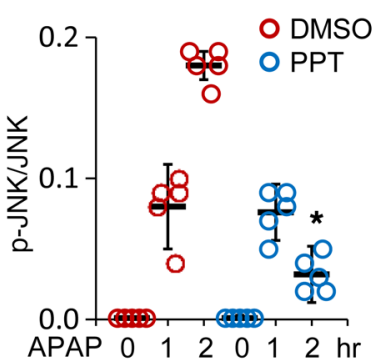
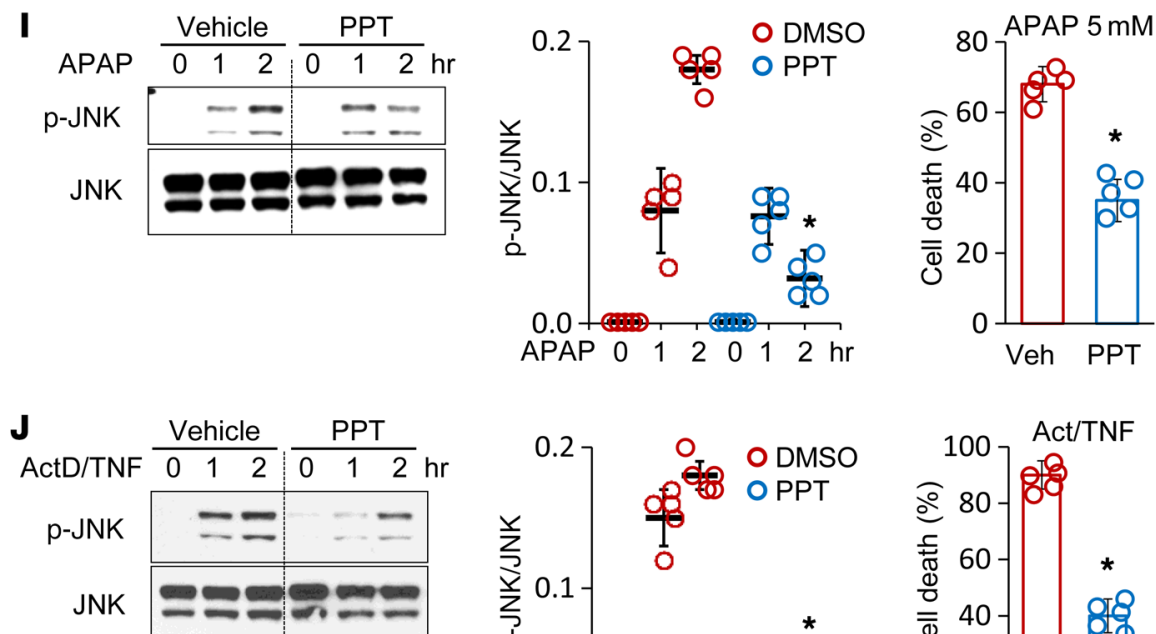
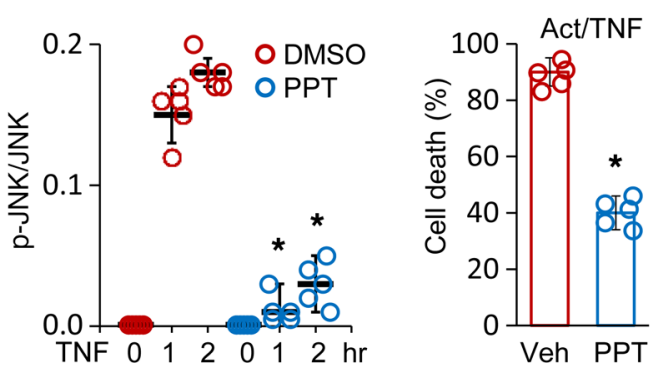
Figure 4. ER $\alpha$ antagonist overcomes resistance to Ad-SAB expression and APAP-induced liver injury in female mice. (A) Female mice received Ad-lacZ or Ad-SAB, and SAB protein levels were analyzed. (B) Female mice were treated with fulvestrant (Fulv) ( 2 or $5 \mathrm{mg} / \mathrm{kg}$ ) once per week for 3 weeks, and $S A B$ expression was determined by immunoblotting. $n=3$ mice/group. ${ }^{*} P<0.05$ versus vehicle (Veh), by 1-way ANOVA with Bonferroni's correction. (C) Female littermates received a total of 3 doses of fulvestrant ( $2 \mathrm{mg} / \mathrm{kg}$ ), 1 dose before and 2 doses ( 1 and 2 weeks) after Ad-lacZ or Ad-SAB $\left(1 \times 10^{9} \mathrm{IU}\right)$ tail-vein injection. SAB expression in isolated mitochondria was determined by immunoblotting. (D) Mice were treated with APAP (300 mg/kg, i.p.). H\&E staining was performed, and serum ALT levels were measured 24 hours later. Scale bars: $100 \mu \mathrm{m} . n=3$ mice/group. ${ }^{*} P<0.05$, versus Ad-lac $Z$, by unpaired, 2 -tailed Student's $t$ test. (E) Male mice were treated with PPT for 2 weeks, and mitochondria $\mathrm{SAB}$ levels were determined. (F and $\mathbf{G}$ ) Male mice were treated with APAP or GaIN/TNF, and H\&E staining was performed and ALT levels determined 6 hours (GalN/TNF) or 24 hours (APAP) later. Scale bars: $100 \mu \mathrm{m} . n=3$ mice/group. ${ }^{*} P<0.05$, versus vehicle, by unpaired, 2 -tailed Student's $t$ test. (H) Male mice were treated with PPT $(5 \mathrm{mg} / \mathrm{kg}$ ) or vehicle for 2 weeks, and $\mathrm{SAB}$ expression in PMHs was determined. (I and J) PMHs from vehicleor PPT-treated male mice were treated with APAP ( $5 \mathrm{mM}$ ) or actinmycin D $(0.5 \mu \mathrm{g} / \mathrm{mL})$ and TNF $(20 \mathrm{ng} / \mathrm{mL})$, respectively. JNK activation in whole-cell lysates was determined by immunoblotting with anti-p-JNK. Immunoblot is representative of 3 separate experiments. ${ }^{*} P<0.05$ versus vehicle, by 1 -way ANOVA with Bonferroni's correction. Cell death was determined with SYTOX Green staining. $n=5$ experiments. ${ }^{*} P<0.05$ versus vehicle, by unpaired, 2-tailed Student's $t$ test. Data are presented as the mean \pm SEM.

ed previously, p-JNK interaction with normal mitochondria inhibits the oxygen consumption rate (OCR) in liver mitochondria from male mice (11). In comparison, female liver mitochondria were resistant to the effect of p-JNK (Figure 2A and Supplemental Figure $3 \mathrm{~A}$ ). In addition, isolated mitochondria from male livers loaded with MitoSOX (detection of $\mathrm{O}_{2}$ ) generated a significantly greater ROS response to p-JNK than was seen in females (Figure 2B). We detected no difference in mitochondrial GSH depletion in male versus female mice 1 or 2 hours after APAP treatment (Supplemental Figure 3B), and the OCR was markedly impaired in the mitochondria of male mice at these time points but was unaffected in female mice (Supplemental Figure 3C). Since we previously showed that the OCR and ROS responses to p-JNK are SAB dependent (11, 12), we hypothesized that there is a sex difference in SAB expression. Indeed, liver mitochondria of female mice expressed $\mathrm{SAB}$ at less than $20 \%$ of the levels detected in their male littermates (Figure 2C) but, interestingly, had similar levels of hepatic Sab mRNA (Supplemental Figure 3D). In addition, SAB protein levels remained constant during the estrus cycle (Supplemental Figure 3E). Since we previously showed that $S a b^{i \mathrm{HHep}}$ male mice are resistant to the toxic effects of APAP and GalN/TNF (11), we hypothesized that the likely explanation for the resistance seen in females in these 2 models was predominantly due to the lower expression of $\mathrm{SAB}$ in female mice. The absence of a sex difference in expression of $S A B$ mRNA suggested posttranscriptional regulation of $S A B$ (see below).

Importantly, we observed a similar sex difference with regard to $\mathrm{SAB}$ expression levels in human liver samples. Samples from men and women were matched for age and BMI, and pre- versus postmenopausal status in women was confirmed by estrogen and follicle-stimulating hormone (FSH) levels. Biopsies were performed for clinical indications so that mild ALT elevations and nonspecific histologic abnormalities were present. Samples for comparison of males and females were paired for age and sex according to menopause status. A representative immunoblot of $S A B$ expression of matched men and premenopausal women (Figure 2D) and a plot of normalized expression of $\mathrm{SAB}$ (Figure 2E) demonstrated markedly lower SAB expression levels in premenopausal women but no sex difference in $\mathrm{SAB}$ expression between postmenopausal women and men and no change in younger versus older men.

Overexpression of SAB increases susceptibility to APAP toxicity in male mice. To test whether the level of $\mathrm{SAB}$ expression is a major determinant of acute liver injury, we next determined the effect of increased $S A B$ expression using adenoviral $S A B$ (Ad-SAB), which contains $S A B$ cDNA (coding region) driven by the CMV promoter. In WT male mice, increasing doses of Ad-SAB resulted in higher $S A B$ expression levels in the liver (Figure $3 \mathrm{~A}$ ), and this was accompanied by enhanced histologic liver necrosis and ALT levels in response to a low dose of APAP $(150 \mathrm{mg} / \mathrm{kg}$ ) (Figure 3B). In addition, an increase in Ad-SAB expression was associated with increased p-JNK 4 hours after APAP (Supplemental Figure 3F), and this correlated with ALT levels in individual mice (Supplemental Figure 3G). However, when we repeated this in female littermates, no increase in SAB expression was detected (Figure 4A), and the mice remained resistant to APAP-induced liver injury (data not shown).

To further assess the importance of SAB expression levels in determining the extent of liver injury, we induced $S A B$ expression with increasing doses of Ad-SAB in SabiHep male mice (Figure 3C). This restored the susceptibility to APAP necrosis (absent in $S a b^{\text {isHep }}$ mice), and led to increased APAP necrosis and serum ALT levels in relationship to increasing viral doses (Figure 3D). To test the possibility that SAB mediates a JNK-independent mechanism of toxicity, we assessed the effect of $\mathrm{SAB}$ overexpression in $J n k 1 / 2^{\text {isHep }}$ mice. Inducible double-knockout of $J n k 1$ and $J n k 2$ with AAV8-TBG-Cre in C57B6J Jnk1/2 ${ }^{f / l l}$ mice (Figure 3E) conferred marked protection against APAP $(600 \mathrm{mg} / \mathrm{kg})$. Since the J substrain is resistant to APAP $(27,28)$, we used a high dose of APAP to ensure the protective effect of $J n k$ deletion (Figure 3F). We found that deletion

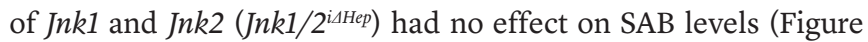
$3 \mathrm{E}$ ) and that concomitant overexpression of SAB (versus control) did not reverse the resistance to APAP in $J n k 1 / 2^{\text {istep }}$ mice (Figure $3 F)$. Therefore, these data indicate that there is no JNK-independent mechanism for the effect of increased SAB overexpression on APAP toxicity. Thus, 2 important conclusions derive from the results shown in Figure 3: (a) the level of SAB expression directly correlates with the extent of liver injury in response to APAP, indicating that the level of $\mathrm{SAB}$ expression is a key checkpoint in determining the severity of liver injury; and (b) JNK is necessary for SAB to increase susceptibility to liver injury and vice versa.

ER $\alpha$ inhibition reverses the resistance to $S A B$ expression and APAP toxicity in female mice. As noted above, we attempted to reverse the resistance of female littermates by overexpressing SAB, but increasing doses of the same Ad-SAB that was used in male mice did not increase hepatic SAB levels in female mice (Figure 4A), nor did it increase susceptibility to APAP or GalN/TNF (data not shown). Estrogen accounts for many downstream sex differences. Estrogen effects may be dependent or independent of estrogen receptors (ERs) (30). The ER is present in 2 isoforms: ER $\alpha$ and ER $\beta$, as well as in a membrane form (GPER). ER $\alpha$ is the main form in liver $(31,32)$ and is expressed at higher levels in females 
A

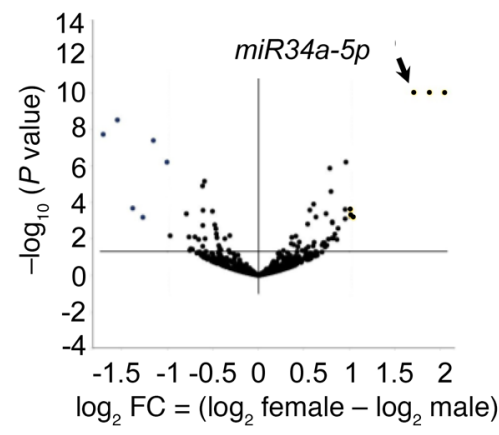

B Mouse Sab mRNA 5' AG CCAGGTACAACGCTGCC $3^{\prime}$ $m i R-34 a-5 p \quad 3^{\prime} \cup \cup G G \cup C G A \cup \cup C \cup G \cup G$ A C G G $5^{\prime}$
C

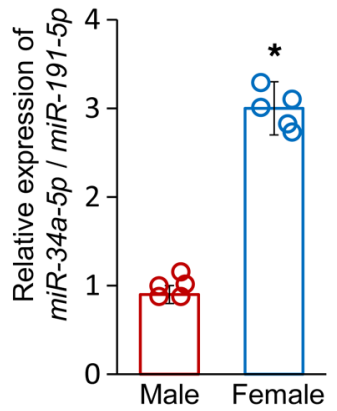

D

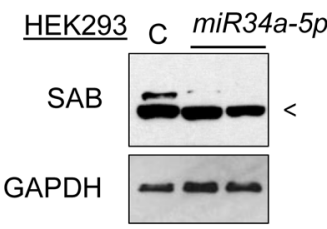

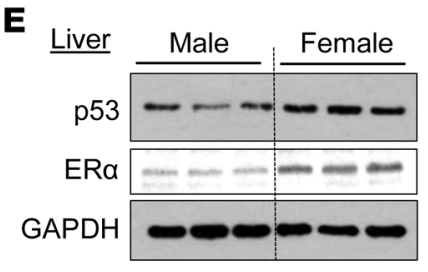

E
$\mathbf{F}$

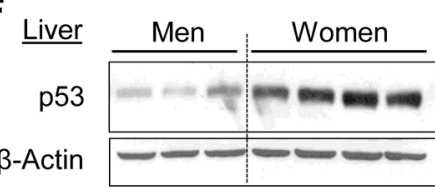

$\beta$-Actin
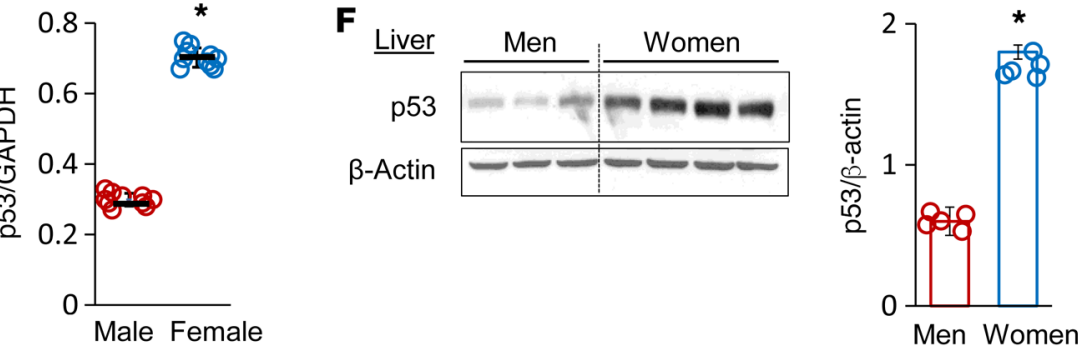
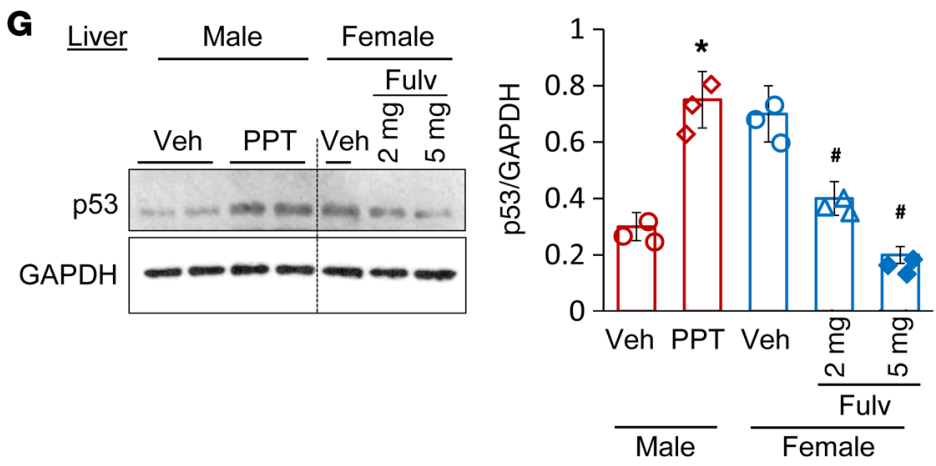

Figure 5. ER $\alpha$ and p53 regulation of miR34a-5p expression. (A) Liver tissue from male and female littermates was snap-frozen in RNAlater RNA stabilizing solution, and miRNA-Seq analysis was performed. $n=3$ mice/group. Volcano plot shows the fold-change (FC) difference in miRNA expression in liver tissue from female and male mice. Sab-targeting miR34a-5p was expressed at a 3.2-fold significantly higher level in female mice than in their male littermates. The $\log _{2}$ fold change (0.59) or (-0.59) is equivalent to a 1.5-fold change in expression in either direction. The arrow points to the dot representing miR34a-5p. (B) miR34a-5p and Sab mRNA alignment. (C) qPCR analysis of miR34a-5p expression. RNA-Seq analysis showed that miR191-5p expression did not differ between male and female mice and was used as a loading control. $n=5$ mice/group, ${ }^{*} P<0.05$ versus male mice, by unpaired, 2 -tailed Student's $t$ test. (D) The miR-34a-5p mimic was transfected into HEK293 cells and cultured for 3 days. Cell survival was the same as that of nontransfected cells (data not shown). SAB levels were determined by immunoblotting with rabbit anti-SAB. For the control (C), cells were transfected with a scrambled oligonucleotide. " $<$ " indicates the nonspecific band. (E and F) Basal levels of p53 and ER $\alpha$ protein expression were determined in male and female littermate mice $\left(n=10\right.$ mice/group; ${ }^{*} P<0.05$ versus male mice, by unpaired, 2-tailed Student's $t$ test) and in human liver tissue from men and premenopausal women ( $n=5$ pairs; ${ }^{*} P<0.05$ versus men, by paired, 2-tailed Student's $t$ test). (C) Male mice were treated with PPT to activate ER $\alpha$, and female mice were treated with fulvestrant to deplete ER $\alpha$. Liver protein extract was examined for p53 expression. Immunoblot is representative of 3 separate experiments. $n=3$ per group. ${ }^{*} P<0.05$ versus vehicle control males; ${ }^{~} P<0.05$ versus vehicle control females, by unpaired, 2 -tailed Student's $t$ test. All data are presented as the mean \pm SEM.

than in males. Both ER $\beta$ and GPER are not detectable in liver (32, 33). To test whether ER $\alpha$ plays an important upstream role in low $\mathrm{SAB}$ expression, we treated female mice with fulvestrant, an ER antagonist that destabilizes the ER $(34,35)$. When female mice were treated with fulvestrant, the levels of ER $\alpha$ decreased and SAB levels increased in a fulvestrant dose-dependent fashion (2 mg/ $\mathrm{kg} ; 5 \mathrm{mg} / \mathrm{kg}$ ) (Figure 4B). PMHs after in vivo treatment with fulvestrant exhibited enhanced $\mathrm{p}-\mathrm{JNK}$ activation in response to APAP
(Supplemental Figure 4A). To further clarify whether the lack of Ad-SAB-mediated expression in females is ER $\alpha$ dependent, we assessed the ability of fulvestrant to unmask Ad-SAB expression in females. Indeed, fulvestrant treatment in female mice before and after Ad-SAB led to increased expression of Ad-SAB in female livers (Figure $4 C$ ) and reversed the resistance of female mice to APAP toxicity (Figure 4D); the females then exhibited markedly increased histologic necrosis and ALT levels. 
$E R \alpha$ activation decreases $S A B$ expression and susceptibility to acute liver injury in male mice. To further define the role of ER $\alpha$ in the mechanism for sex difference in SAB levels, we treated male mice in vivo with a well-characterized potent and specific ER $\alpha$ chemical ligand activator, propylpyrazole triol (PPT), which does not activate $\operatorname{ER} \beta(36,37)$. Indeed, PPT treatment for 5 days markedly decreased expression of SAB protein (Figure $4 \mathrm{E}$ ) but not that of Sab mRNA (Supplemental Figure 4B). PPT markedly protected male mice from both APAP and TNF/GalN toxicity as reflected in histologic injury and ALT levels in vivo (Figure 4, F and G).

To further assess the cell-autonomous effect of the ER $\alpha$ agonist PPT, we isolated hepatocytes from males after in vivo treatment with PPT or vehicle. We then exposed the PMH cultures to APAP or ActD/TNF. In both models, PPT pretreatment in vivo with resulting SAB depletion (Figure $4 \mathrm{H}$ ) protected against $\mathrm{PMH}$ cell death accompanied by inhibition of sustained JNK activation in response to APAP or ActD/TNF (Figure 4, I and J). Furthermore, PPT pretreatment of male mice in vivo protected against PMH cell death due to tunicamycin (Supplemental Figure 4C) or palmitic acid (Supplemental Figure 4D), and decreased palmitic acid induced sustained JNK activation (Supplemental Figure 4E). In addition, treatment of normal male hepatocytes with an ER $\beta$ agonist (DPN) or androgen receptor agonist (BMS) did not affect $\mathrm{SAB}$ expression (Supplemental Figure $4 \mathrm{~F}$ ). Therefore, the findings shown in Figure 4 suggest that SAB repression is mediated by $\mathrm{ER} \alpha$ and that activation of $\mathrm{ER} \alpha$ consequently leads to suppression of sustained JNK activation and acute hepatic and cellular injury in males, whereas inactivation of ER $\alpha$ increases SAB expression and $\mathrm{p}$-JNK activation in females and unmasks the ability of Ad-SAB to restore susceptibility to liver injury in females.

miR34a targets $S a b m R N A$. As noted above, when we attempted to overexpress Ad-SAB in female mice, no increase in SAB expression was observed, and the mice remained resistant, with no increase in APAP-induced liver injury. The basal resistance of female mice to the expression of Ad-SAB strongly suggested interference with SAB expression at the posttranscriptional level. The results in male mice treated with the ER $\alpha$ agonist and in female mice treated with the ER degrader supported the importance of $\mathrm{ER} \alpha$ in upstream regulation of SAB. Furthermore, despite the large difference in SAB protein expression, we observed no difference in hepatic $S a b$ mRNA levels in female mice versus male mice. Taken together, the findings led us to the hypothesis that $\mathrm{ER} \alpha$, either directly or indirectly via an intermediate, may regulate the expression of a miRNA that is targeting the $S A B$ mRNA coding region of the Ad-SAB cDNA. Therefore, we performed miRNA-Seq analysis of liver samples from untreated male and female littermates. The results are shown in Figure 5A as a volcano plot comparing littermate females with males. Three miRNAs stood out as highly significant, as reflected in greater than 3-fold higher levels in female mice. However, only 1 miRNA targets $S a b$ mRNA, i.e., miR34a-5p, which is predicted to target the coding region (Figure $5 \mathrm{~B}$ ); since Ad-SAB cDNA does not contain a 3 '-untranslated sequence, we expected that a miRNA would have to target the coding region. The sex difference in miR34a-5p expression was confirmed by quantitative PCR (qPCR) (Figure 5C). To confirm the capability of miR34a-5p to repress SAB levels, we induced expression of miR34a-5p in HEK293 cells and observed a decrease in $\mathrm{SAB}$ protein expression (Figure 5D).
ER $\alpha$ regulates $p 53$, which increases $m i R 34 a-5 p$. Since miR34a-5p was a likely candidate for repression of $S a b$ mRNA translation, we next addressed the link between ER $\alpha$ and miR34a-5p. Two important facts were derived from prior publications: miR34a expression is transcriptionally regulated by $\mathrm{p} 53$ in other contexts, and ER $\alpha$ is known to regulate p53 expression (38). Therefore, we examined p53 levels in males versus females and found significantly higher levels of hepatic p53 in female liver, in both mice and premenopausal women (Figure 5, E and F). Thus, we found a sex-dependent inverse relationship between p53 and SAB (Figure 1, G and $\mathrm{H}$, and Figure 5, E and F). We therefore determined the effect of ER $\alpha$ agonist (PPT) treatment in male mice and ER $\alpha$ antagonist (fulvestrant) treatment on p53 expression in female mice. We found that hepatic p53 was increased after PPT treatment of male mice and dose dependently decreased after fulvestrant treatment of female mice (Figure 5G). These data confirmed the regulation of p53 expression by estrogen.

After confirming the link between ER $\alpha$ and p53, we assessed the effect of p53 knockdown in vivo using a p53 antisense oligonucleotide (ASO) versus a scrambled ASO control. Knockdown of p53 increased SAB expression levels in both male and female mice. Interestingly, p53 and SAB levels in females after knockdown of p53 were comparable to the basal levels in male controls (Figure 6A). As expected, p53 knockdown led to decreased miR34a-5p levels in both male and female littermates (Figure 6B), which was accompanied by increased SAB levels (Supplemental Figure 5A). We also confirmed that p53-ASO (ASO no. 2) targeting a different locus of $p 53$ mRNA also enhanced APAP toxicity (Supplemental Figure 5B). As seen with SAB, the level of miR34a-5p in females after p53 knockdown was nearly identical to the basal level in the male control. Conversely, to determine whether increased p53 expression and activation would repress SAB, we treated HepG2 cells with a sublethal dose of doxorubicin (induction of p53 by DNA damage) and the HDAC inhibitor trichostatin A (increased acetyl-p53), as previously described (39-41), and observed that $\mathrm{SAB}$ expression was markedly decreased after treatment, confirming that upregulation of $\mathrm{p} 53$ leads to downregulation of SAB (Supplemental Figure 5C).

We analyzed SIRT1 expression, as it is known to be repressed by miR34a-5p (42). Also, SIRT1 can deacetylate p53 and somewhat inhibit its activity $(43,44)$. However, unexpectedly, we found that the basal expression levels of both SIRT1 and acetyl-p53 were greater in female mouse liver, despite the lower SAB expression (Figure 6A and Supplemental Figure 5D). Also, women exhibited higher hepatic SIRT1 levels (Supplemental Figure 5E). However, despite the differences in basal levels, p53 knockdown decreased $m i R 34 a$ and increased SAB and SIRT1 protein expression in both males and females (Figure 6, A and B).

To further confirm the key role of p53 in ER $\alpha$-mediated repression of SAB, we examined PMHs from male mice treated with PPT after in vivo treatment with p53-ASO. Scrambled ASO pretreatment did not alter the repression of SAB by the ER $\alpha$ agonist PPT (Figure 6C). PPT treatment of male control hepatocytes increased p53 levels, directly demonstrating regulation of p53 expression by activation of ER $\alpha$ (Figure 6C). However, p53-ASO pretreatment and efficient knockdown of p53 prevented PPT-mediated repression of SAB expression (Figure 6C). Importantly, p53 knockdown 

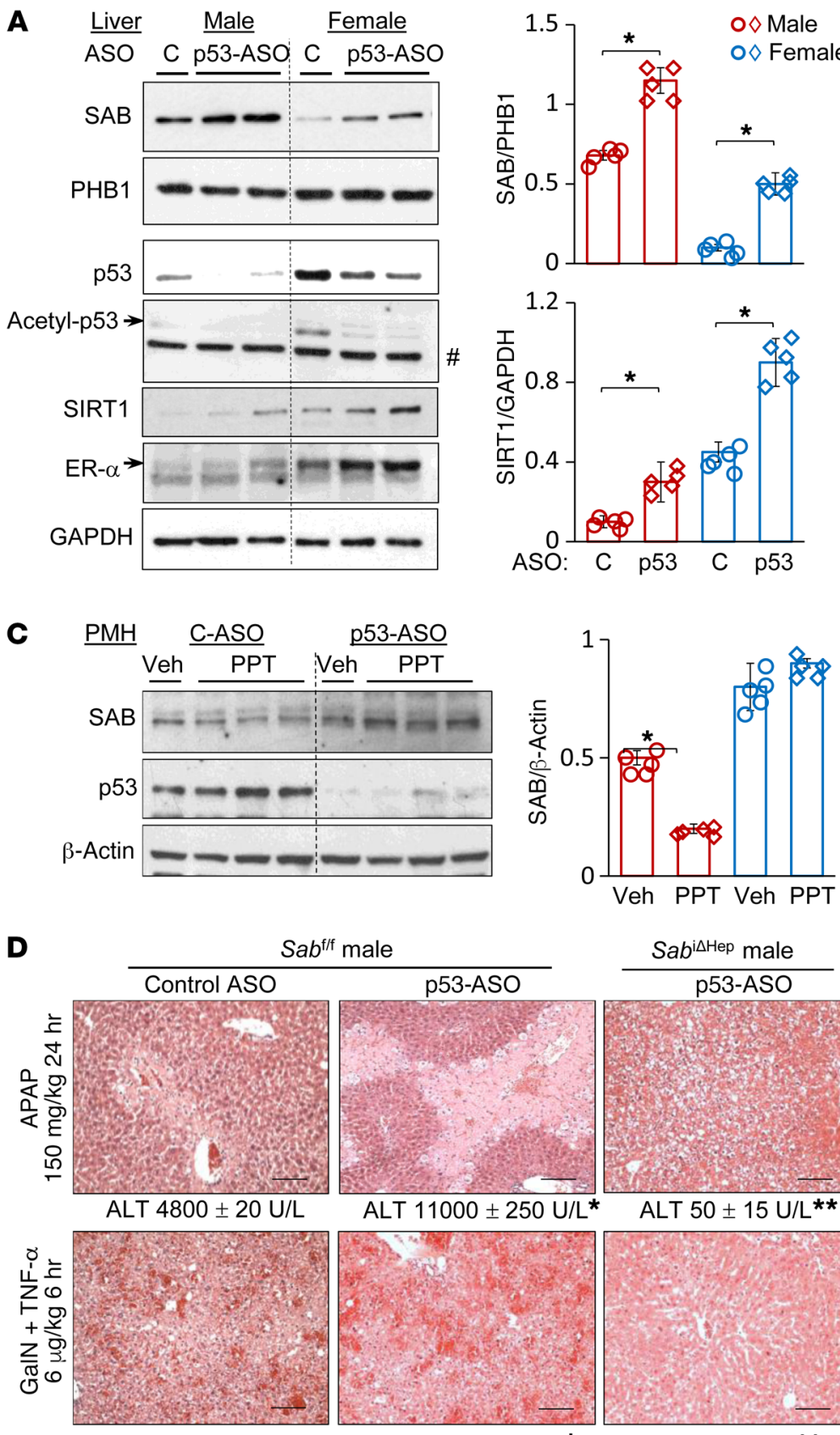

$\mathrm{ALT} 3800 \pm 23 \mathrm{U} / \mathrm{L}$

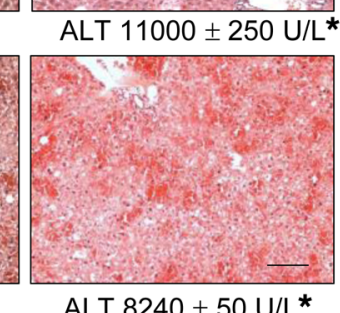

E
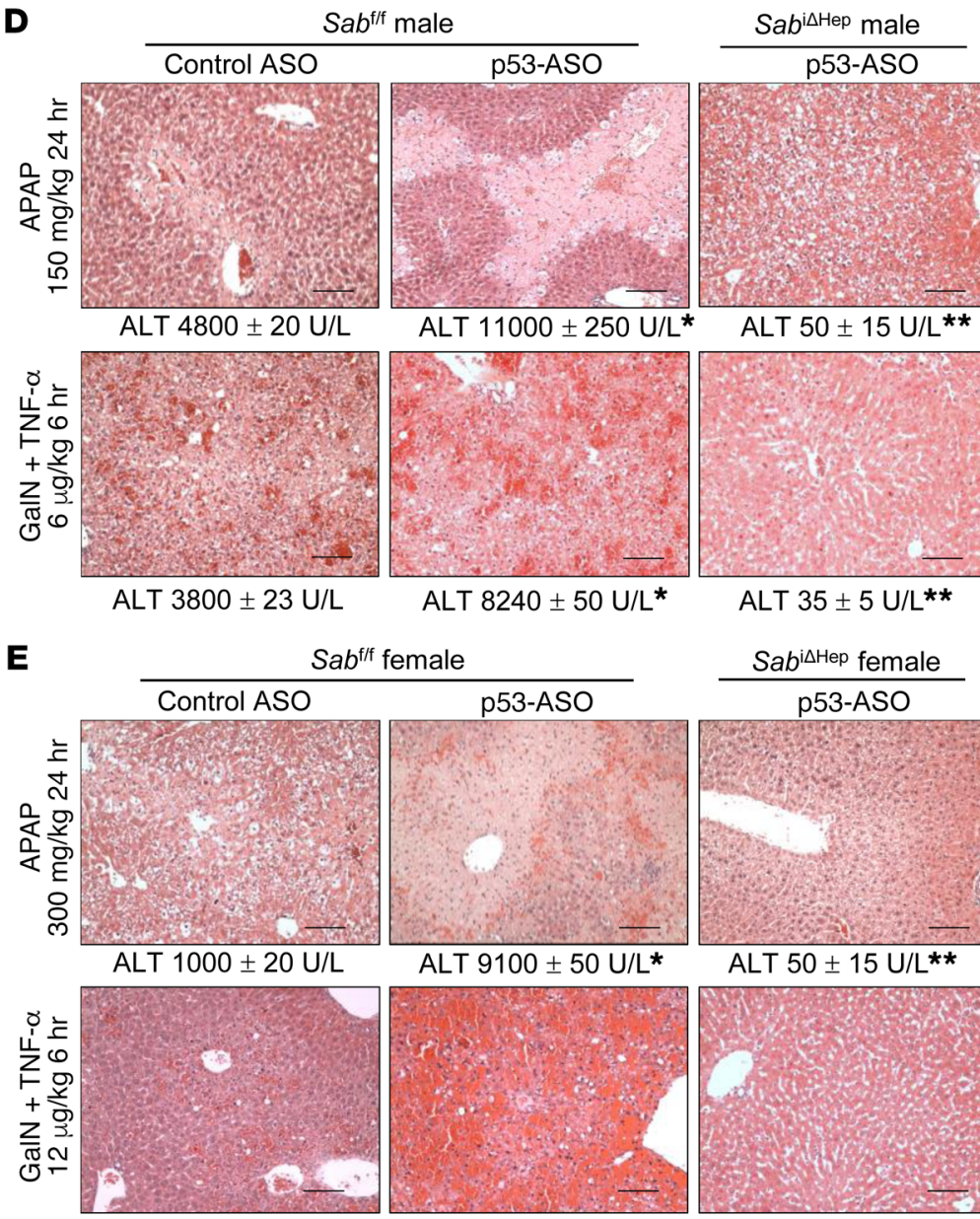

$\mathrm{ALT} 420 \pm 50 \mathrm{U} / \mathrm{L}$
$\mathrm{ALT} 8240 \pm 50 \mathrm{U} / \mathrm{L}^{*}$
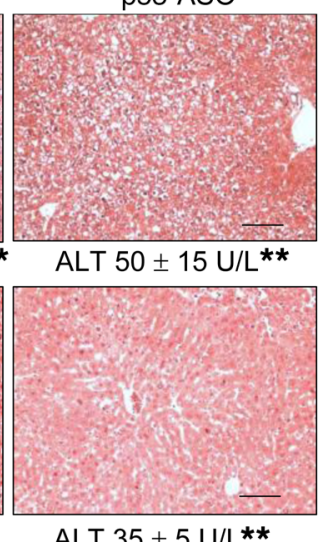

$\operatorname{ALT} 35 \pm 5 \mathrm{U} / \mathrm{L}^{\star *}$
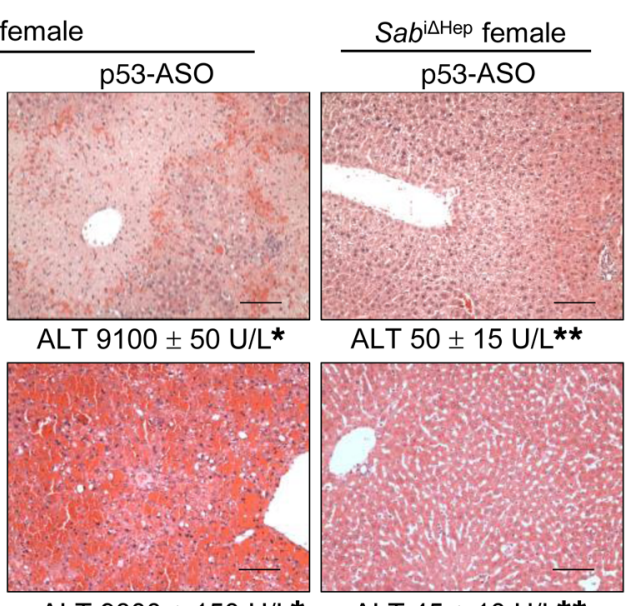

ALT $9800 \pm 150 \mathrm{U} / \mathrm{L}^{\star} \quad$ ALT $45 \pm 10 \mathrm{U} / \mathrm{L}^{\star *}$
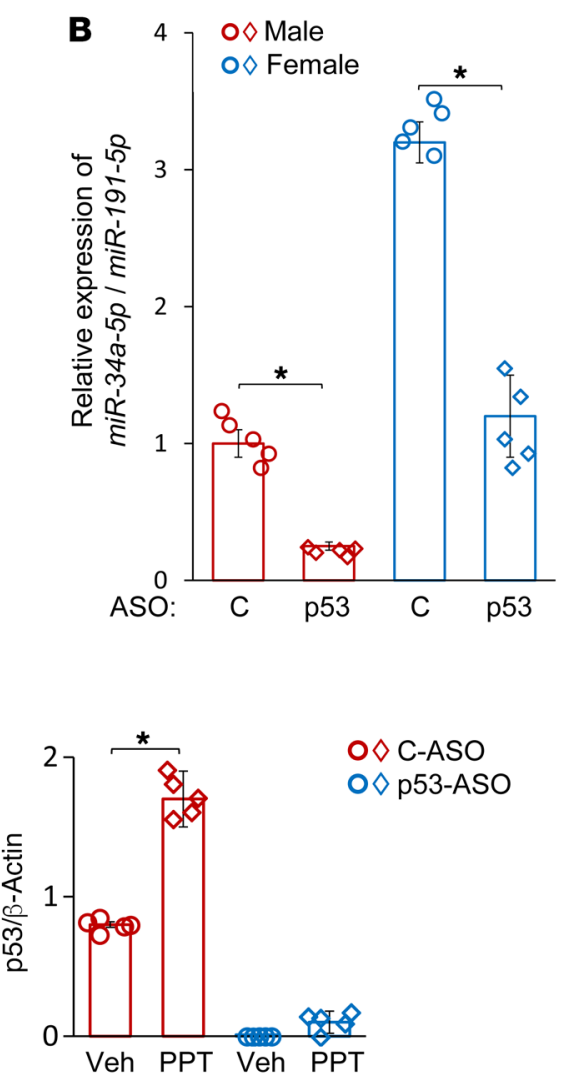

Figure 6. p53 modulates hepatotoxicity through SAB expression. Ten- to twelve-week-old littermate male or female mice received 7 doses of a scrambled control or p53-ASO (50 mg/kg, i.p.) over a 2-week period. (A and B) $\mathrm{SAB}$ and PHB1 expression levels were determined in the mitochondrial fraction. p53, acetyl-p53, SIRT1, ER- $\alpha$, and CAPDH levels were determined in whole-liver extracts. Immunoblot is representative of 3 separate experiments. Expression of miR-34a-5p was quantitated by qPCR. The pound sign indicates the nonspecific band, and the arrow indicates the specific band for acetyl-p53. $n=5$ mice/ group. ${ }^{*} P<0.05$ versus control ASO, by unpaired, 2 -tailed Student's $t$ test. Data are presented as the mean \pm SEM. (C) Scrambled control- or p53-ASO-treated PMHs from male mice were treated with DMSO (Veh) or PPT $(10 \mu \mathrm{M})$ for 2 days. Immunoblot is representative of 3 separate experiments. $n=5$ mice/group. ${ }^{*} P<0.05$ versus vehicle control, by unpaired, 2-tailed Student's $t$ test. Data are presented as the mean \pm SEM. (D and E) Scrambled control- or p53-ASO-treated Sab $b^{f / f l}$ and Sabistep male and female mice were fasted overnight and treated with APAP (150 or 300 $\mathrm{mg} / \mathrm{kg}$, i.p.) or GaIN ( $800 \mathrm{mg} / \mathrm{kg}$, i.p.) and TNF (6 or $12 \mu \mathrm{g} /$ $\mathrm{kg}$, i.p.), and 6 hours (GalN/TNF) and 24 hours (APAP) later, liver histology was performed and serum ALT levels determined. Representative H\&E-stained images are shown. Scale bars: $100 \mu \mathrm{m} . n=3$ mice in the control ASO-treated group; $n=5$ mice in the p53-ASO-treated groups. ${ }^{*} P<0.05$ versus the control ASO-treated group; ${ }^{* *} P<0.05$ versus the p53-ASO-treated Sab ${ }^{f / f l}$ group, by unpaired, 2-tailed Student's $t$ test. Data are presented as the mean \pm SD. 
A
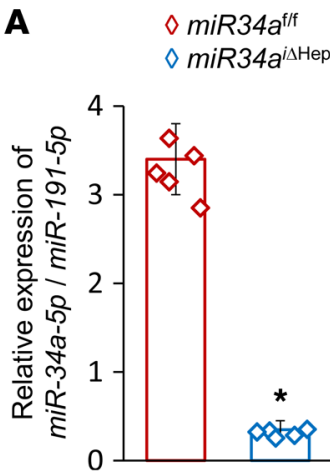

B

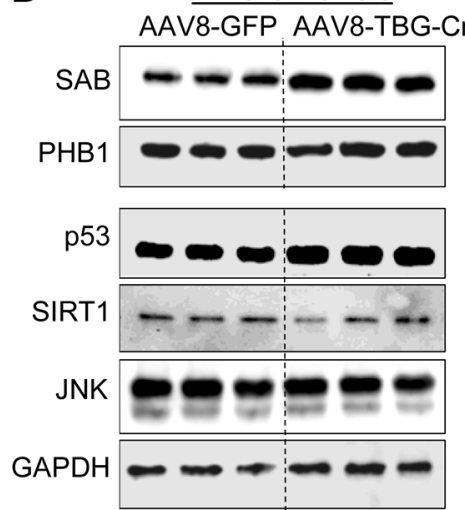

OAAV8-GFP OAAV8-TBG-Cre
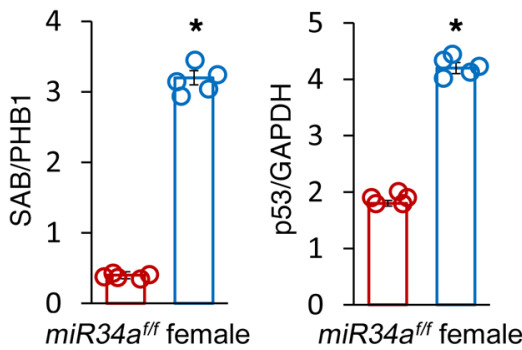

C

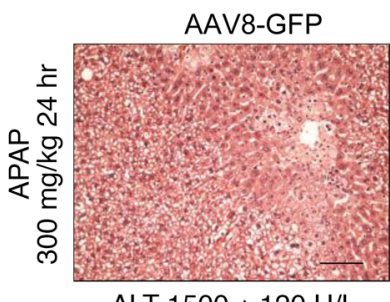

ALT $1500 \pm 120 \mathrm{U} / \mathrm{L}$

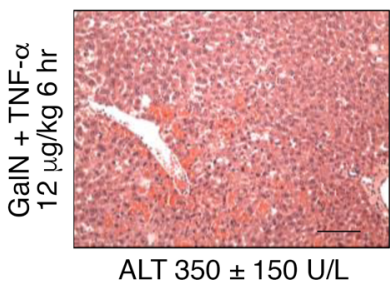

ALT $350 \pm 150 \mathrm{U} / \mathrm{L}$ $\underline{\text { miR34a } a^{f / f} \text { female }}$

AAV8-TBG-Cre
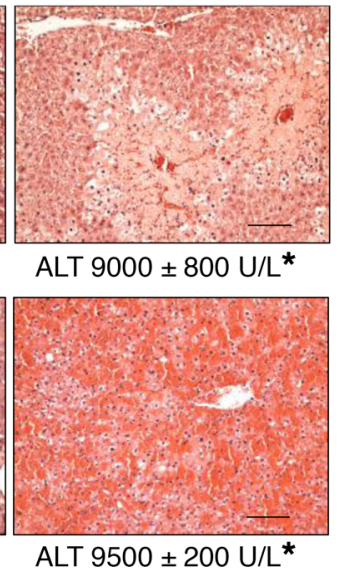

D

miR34afff female

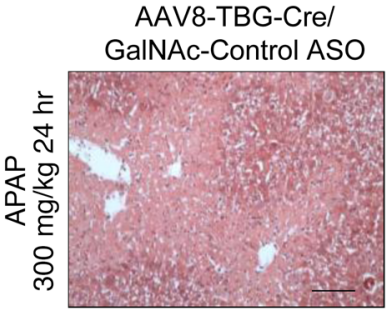

ALT $10000 \pm 300$ U/L

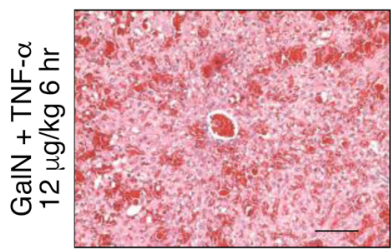

ALT $9100 \pm 250 \mathrm{U} / \mathrm{L}$
AAV8-TBG-Cre/

GalNAc-Sab ASO

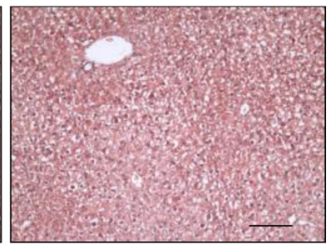

$\mathrm{ALT} 40 \pm 5 \mathrm{U} / \mathrm{L}^{*}$

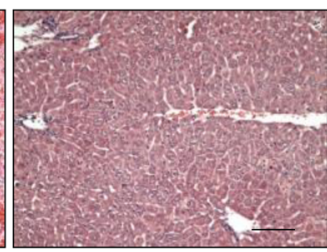

ALT $52 \pm 5 \mathrm{U} / \mathrm{L}^{\star}$

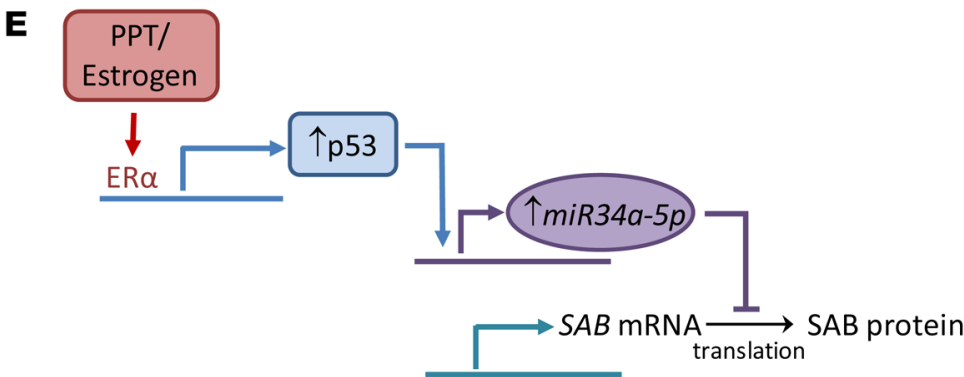

Figure 7. miR34a-5p modulates SAB expression and hepatotoxicity. miR34a $a^{f / f l}$ female mice received AAV8-TBG-GFP or AAV8-TBG-Cre via tail-vein

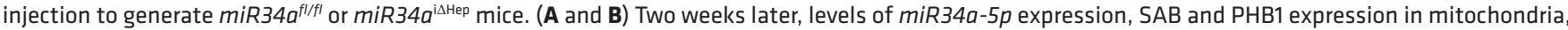
and p53, SIRT1, and GAPDH expression in liver extract were determined. $n=5$ mice/group. ${ }^{*} P<0.05$ versus the AAV8-GFP group, by unpaired, 2-tailed Student's $t$ test. Data are presented as the mean \pm SEM. (C) Overnight-fasted mice received APAP or GaIN/TNF, and liver histology was performed and serum ALT levels determined 6 hours (GaIN/TNF) and 24 hours (APAP) later. Representative H\&E-stained images are shown. Scale bars: $100 \mu \mathrm{m}$. $n=5$ mice/ group. ${ }^{*} P<0.05$ versus the AAV8-GFP group, by unpaired, 2 -tailed Student's $t$ test. Data are presented as the mean $\pm \mathrm{SD}$. (D) $\mathrm{miR}^{\mathrm{i}} \mathrm{B} a^{\mathrm{i} \Delta \mathrm{Hep}}\left(\mathrm{miR} 34 a^{\mathrm{fl} / \mathrm{fl}}\right.$ plus AAV8-TBG-Cre) female mice received GaINAc-control or Sab-ASO for 2 weeks to deplete hepatocellular SAB expression. Overnight-fasted mice then received APAP or GaIN/TNF, and liver histology was performed and serum ALT levels determined 6 hours (GaIN/TNF) and 24 hours (APAP) later. Representative $\mathrm{H} \& \mathrm{E}$-stained images are shown. Scale bars: $100 \mu \mathrm{m} . n=5$ mice/group. ${ }^{*} P<0.05$ versus GalNAc-control-ASO, by unpaired, 2 -tailed Student's $t$ test. Data are presented as the mean \pm SD. (E) Schematic presentation of the $E R \alpha / p 53 / m i R 34 a-5 p$ axis of regulation of SAB expression.

did not unmask an alternative mechanism of ER $\alpha$-mediated PPT repression of $\mathrm{SAB}$, as the increase in $\mathrm{SAB}$ expression after $p 53$ knockdown was not inhibited by PPT activation of ER $\alpha$ (Figure 6C). Therefore, ER $\alpha$ regulates p53 expression, which increases miR34a and leads to repression of SAB.
We recently reported that there was an inverse relationship between acute liver injury due to APAP in male mice with p53 inhibition or knockdown, leading to increased p-JNK, and toxicity, whereas p53 activation led to decreased p-JNK and protection (45). Therefore, we extended our studies by examining the effect 
of p53 knockdown on liver injury in female mice treated with APAP or GalN/TNF. In both injury models, p53 knockdown led to increased injury in both male and female $S a b^{f / f l}$ controls, which was most striking in female mice and led to toxicity comparable to that seen in male mice (Figure 6, D and E). Increased APAP toxicity in female $m i R 34^{i \text { iHep }}$ mice was accompanied by enhanced p-JNK at the early time point (Supplemental Figure 6A). Of note, the enhanced JNK activation after APAP in female miR34 ${ }^{\mathrm{i} \Delta \mathrm{Hep}}$ mice was accompanied by impaired GSH recovery (Supplemental Figure 6B), whereas male mice treated with the miR34a-5p mimic (decreased SAB and p-JNK) showed enhanced GSH recovery (Supplemental Figure 6C). These findings support the conclusion that the difference in GSH recovery is not an intrinsic sex-dependent phenomenon but is secondary to the effect of SAB-JNK and the severity of injury. In addition, the marked resistance of $S a b^{\text {iАHep }}$ male and female mice to APAP and GalN/TNF was not overcome by $p 53$ knockdown (Figure 6, D and E), indicating that the worsening of liver injury after p53 inhibition in both models and the sex are dependent on SAB expression and not due to an alternative p53-regulated protective mechanism.

Expression of miR34a-5p directly determines the severity of acute liver injury. Having established a pathway from $\mathrm{ER} \alpha$ to $\mathrm{p} 53$ to $\mathrm{miR} 34 \mathrm{a}$ $5 p$ to $\mathrm{SAB}$ repression, further evidence was required to conclusively link miR34a-5p to protection from liver injury. Therefore, we treated male mice with the Ad-miR34a-5p mimic, which led to increased miR34a-5p levels (Supplemental Figure 7A) and was accompanied by a marked decrease in SAB protein expression to levels that were low but comparable to those in untreated females (Supplemental Figure 7B). However, $S a b$ mRNA levels were not changed by the Ad-miR34a-5p mimic (Supplemental Figure 7C). Under these conditions, the miR34a-5p mimic was successful in markedly protecting male mice against APAP- and GalN/TNF-induced liver injury (histologic injury and serum ALT) compared with the controls (Supplemental Figure 7D). In contrast, we compared female $m i R 34 a^{f / f l}$ mice treated with AAV8-TBG-GFP (control) or AAV8-TBG-Cre. The miR34a $a^{\text {i } \Delta \text { Hep }}$ females exhibited extremely low miR34a levels (Figure 7A). Inducible knockout of miR34a led to increased SAB protein levels (Figure 7B). Interestingly, $m i R 34 a^{\mathrm{i} \Lambda \mathrm{Hep}}$ mice had increased p53 levels, a finding consistent with previous reports that miR34a represses p53 in HepG2 cell (46), but increased p53 conferred no protection against liver injury in the $m i R 34 a^{\text {is Hep }}$ mice. As expected, SIRT1 levels were decreased by the miR34a-5p mimic, but we observed no increase in $m i R 34 a^{\mathrm{i} \Lambda \mathrm{Hep}}$ mice (Figure 7B and Supplemental Figure 7B). In both APAP- and GalN/TNF-induced injury models, miR34 $a^{\text {isHep }}$ mice exhibited markedly enhanced liver injury (histologic injury and serum ALT) (Figure 7C). In fact, the injury in female $m i R 34 a^{f / f l}$ mice treated with AAV8-TBG-Cre (Figure 7C) was comparable to that seen in APAP- or GalN/TNF-treated male controls (Ad-miR-GFP) (Supplemental Figure 7D). $m i R 34 a^{\text {is Hep }}$ female mice express increased SAB protein but not Sab mRNA (Supplemental Figure 7E), again consistent with miR34a repression of $S a b$ mRNA translation into protein. Finally, to test whether the derepression of $S a b$ in $m i R 34 a^{\text {iAHep }}$ mice is the mechanism for enhanced liver injury, we knocked down Sab in female $m i R 34 a^{\mathrm{i} \Lambda \mathrm{Hep}}$ mice using GalNAc-Sab ASO compared with GalNAc-scrambled control ASO (Figure 7D). The GalNAc derivative increases ASO potency and selectively targets hepatocytes through the asialoglycoprotein receptor (47). We found that treatment with the GalNAc-Sab ASO in vivo depleted SAB in hepatocytes but had no effect on SAB in nonparenchymal cells (Supplemental Figure $7 \mathrm{~F}$ ). Treatment of female miR34 $a^{\text {i Hep }}$ mice with scrambled ASO showed no effect on SAB levels or the enhanced injury response to APAP or GalN/TNF, whereas the GalNAc-Sab ASO markedly decreased hepatocyte $\mathrm{SAB}$ expression and prevented enhanced liver injury in the miR34 $a^{\text {i } \mathrm{AHep}}$ female mice, as seen with the parent Sab ASO (Figure 7D and Supplemental Figure 7, G and H).

\section{Discussion}

The JNK-SAB-ROS activation loop plays a critical role in sustaining JNK activation (9). Prolonged JNK activation can promote cell death by phosphorylation of transcription factors, which increase expression of BH3 members of the BCL2 family (e.g., PUMA) $(25,48)$, or by direct activation of proapoptotic BCL2 family members (e.g., BAX, BID) or inactivation of antiapoptotic proteins (e.g., MCL-1, BCL- $\mathrm{X}_{\mathrm{L}}$ ) (21-24). SAB plays a pivotal role in mediating mitochondrial ROS production $(11,12)$, but very little is known about the regulation of SAB expression. We previously reported that $S a b$ knockdown or hepatocyte-specific inducible deletion of $S a b$ markedly protects against liver injury in models in which injury is JNK dependent $(10,11)$. Thus far, our understanding of the role of SAB is based on its absence versus its presence. However, it is important to understand whether $\mathrm{SAB}$ expression is regulated under certain physiological conditions and whether this influences the susceptibility to and severity of liver injury. We approached this question by examining SAB expression levels in male versus female mice, because it has been reported that females are resistant to APAP toxicity and exhibit lower sustained JNK activation and mitochondrial translocation but show no differences in expression of total JNK compared with males. Therefore, we considered this a potentially informative scenario in which to address the role of SAB expression. In addition, we examined the restoration of SAB expression to different levels in $S a b^{\text {ìHep }}$ mice to examine the relationship of the level of $\mathrm{SAB}$ expression and the severity of liver injury. We confirmed the resistance of female mice to APAP injury. However, the females were also resistant to the toxicity of GalN/TNF in vivo, another JNK-dependent acute liver injury model, which is independent of drug metabolism and GSH status. In addition, cultured PMHs from female mice were resistant to apoptotic cell death from tunicamycin and palmitic acid. These findings strengthened the hypothesis that there might be a difference in basal mitochondrial $\mathrm{SAB}$ expression contributing to the resistance of female mice to liver injury compared with male mice. SAB is the binding target and substrate of p-JNK (49). In support of the possibility that there may be a sex difference in SAB expression, we observed that liver mitochondria from female mice were less susceptible to $\mathrm{p}$-JNK plus ATP-induced inhibition of respiration and enhancement of ROS production. Indeed, SAB protein levels in mitochondria from female mice were less than $20 \%$ of the male levels. Importantly, human liver with near-normal histology revealed a similar sex difference in $\mathrm{SAB}$ expression. The correlation between resistance to liver injury with low $S A B$ levels led us to address the mechanism and importance of decreased SAB expression in females and to gain new insight into the regulation and importance of SAB. 
To elucidate the mechanism for SAB repression in females, we assessed the effect of activating ER $\alpha$ in males and inactivating $E R \alpha$ in females. ER $\alpha$ is the major target of estrogens and is expressed at high levels in females. ER $\alpha$ agonist treatment of males lowered SAB expression and ER $\alpha$ antagonist increased $\mathrm{SAB}$ expression in females. In males, the activation of ER $\alpha$ was accompanied by resistance to acute liver injury due to APAP or GalN/TNF, whereas the opposite was observed in females treated with an $\mathrm{ER} \alpha$ antagonist.

To address the importance of SAB expression levels in determining the severity of liver injury in males as well as sex difference in susceptibility, we first induced overexpression of SAB in WT male mice and $S a b^{\text {i } \triangle \mathrm{Hep}}$ mice using Ad-SAB cDNA and observed a direct relationship between the level of SAB expression and liver injury due to APAP. Though most research in liver injury models supports a pivotal role of JNK in mediating hepatocellular death, a recent study found that mice with liver-specific embryonic knockout of Jnk1 that were crossed with global Jnk2-knockout mice exhibited increased APAP-induced liver injury, indicating that JNK might be protective (50). Since this study and ours used different approaches (embryonic versus inducible) to delete Jnk1/2, their findings are not directly comparable. Recently, Zoubek et al. (51) found a hepatocyte-specific protective role of JNK2 in ibuprofen-induced acute liver failure model (worsened with JNK2 knockdown). Therefore, studies of the relative contribution of JNK1 versus JNK2 in the models we have used will be of great interest and are planned for the future. However, the work of Cubero et al. raises an important question relevant to our work on SAB as to whether the unequivocal participation of SAB in liver injury might be due to an alternative JNK-independent mechanism for $\mathrm{SAB}$ dependence in acute liver injury. To address this question, we crossed $J n k 1^{f / f l}$ and $J n k 2^{f / f l}$ mice (C57B6J strain) and injected AAV8-TBG-Cre into 2-month-old double-floxed adult mice to induce combined hepatocyte $J n k 1$ and 2 deletion in the livers of the adult males. Deletion of both Jnk1 and Jnk2 in these mice caused a striking resistance to APAP but had no effect on SAB expression. We then overexpressed SAB in the Jnk1/2 $2^{\text {isHep }}$ mice but found no restoration of susceptibility to APAP toxicity. These findings confirm that enhanced APAP toxicity after increased SAB expression is JNK dependent. Furthermore, the findings indicated that it is unlikely that a JNK-independent mechanism accounts for the role of SAB in liver injury.

Studies by the Chambers laboratory are also consistent with the importance of the levels of SAB expression in cell injury. Overexpression of SAB sensitized both $\mathrm{H} 9 \mathrm{c} 2$ cardiac cells, and $\mathrm{HeLa}$ cells to toxicity from drugs such as imatinib and chemotherapy drugs $(52,53)$. Furthermore, they demonstrated that in a panel of ovarian cancer cells, the levels of SAB expression correlated with the chemotoxicity: SAB levels correlated with high susceptibility, and overexpression of $\mathrm{SAB}$ in a chemoresistant cell line increased sensitivity to the chemotherapy drug (54). These studies and the present work indicate that the level of mitochondrial SAB dictates the magnitude of JNK activation and signaling under toxic stress. Of note, Paudel et al. (54) found that ovarian cancer cells with high SAB expression were primed for apoptosis, as reflected in decreased prosurvival BCL-2 proteins and increased BH3-only proteins on mitochondria. These findings in cultured cancer cells differ from our results in the liver showing no changes in BCL-2 proteins, probably because under basal conditions, SAB overexpression does not activate JNK or c-JUN in vivo in the liver.

Having established the relationship between SAB expression and the severity of liver injury in male mice, we then attempted to overexpress $\mathrm{SAB}$ in female mice, expecting to demonstrate that overexpression of $\mathrm{SAB}$ in females would eliminate the sex difference. However, we observed no increase in either SAB levels or susceptibility to liver injury after administering Ad-SAB. Since the $S A B$ cDNA is derived from $S A B$ mRNA corresponding to the coding region of the $S A B$ gene, a posttranscriptional mechanism for repression of $S A B$ was suspected. Indeed, a miRNA sequence comparison between male and female littermates under basal conditions revealed several miRNAs that were much more highly expressed in female livers. Among these, only miR34a-5p was predicted to target $S a b$ mRNA (at the coding region corresponding to exon 4). When $\mathrm{ER} \alpha$ was inhibited by fulvestrant in female mice, the Ad-SAB-mediated expression of SAB was unmasked, and females became sensitized to APAP- and GalN/TNF-induced liver injury. Since the sex difference in $\mathrm{SAB}$ protein expression was not accompanied by a difference in $S a b$ mRNA levels, a repression of translation of $S a b$ mRNA was strongly indicated.

miR34a-5p is known to be transcriptionally regulated by p53 $(46,55,56)$. Interestingly, we found that p53 levels were significantly higher in liver tissue of female mice and women compared with that of male mice and men. Furthermore, the ER $\alpha$ agonist increased p53 expression in males and the ER $\alpha$ antagonist decreased $\mathrm{p} 53$ in females, confirming that $\mathrm{ER} \alpha$ regulates $\mathrm{p} 53$, which then increases miR34a-5p expression. We recently reported that p53 had a protective effect against APAP in male mice (45). We now show that p53 induces the expression of $m i R 34 a-5 p$, which represses $\mathrm{SAB}$ and protects against JNK activation and liver injury. Although JNK is known to phosphor- activate p53 (57-59), the repression of SAB inhibits sustained JNK activation and therefore would minimize an effect on p53-induced cell death.

The importance of miR34a-5p was directly confirmed by expression of a mimic in male mice, which led to a resistance to liver injury, and by deletion of the miR34a gene in the liver of female mice, which led to greatly increased susceptibility to liver injury. These effects correlated with the levels of sustained JNK activation. The $m i R 34 a^{\mathrm{i} \Lambda \mathrm{Hep}}$ mice exhibited derepression of SAB and enhanced acute liver injury, which were abrogated by $S a b$ knockdown, leading us to conclude that a major factor in the influence of miR34a on acute APAP- and GalN/TNF-induced liver injury is its effect on $\mathrm{SAB}$ protein expression and that $\mathrm{SAB}$ is indispensable for APAP and GalN/TNF hepatotoxicity. Though the miR34a-5p mimic decreased $\mathrm{SAB}$ protein expression and the knockout of $m i R 34 a$ increased SAB protein expression, neither one changed $S a b$ mRNA levels, which further supports the hypothesis that miR34a inhibits the translation of $S a b$ mRNA rather than causing degradation. Importantly, treatment of $m i R 34 a^{i \Delta \mathrm{Hep}}$ mice with GalNAc-Sab-ASO, which caused hepatocyte-specific efficient knockdown of $S a b$, prevented the enhanced liver injury, conclusively indicating that miR34a exerts its effect on hepatotoxicity through regulation of SAB.

In parallel to the findings in mice, human liver samples showed a similar reciprocal relationship between p53 and SAB, with premenopausal women exhibiting high p53 and low SAB lev- 
els and men exhibiting low p53 and high SAB levels. Although this suggests that our findings may be relevant to humans, we could find no conclusive evidence that the decreased susceptibility of female mice to acute liver injury does or does not translate to humans because of a paucity of data comparing the susceptibility of males and females. A study using male and female hepatocytes exposed to different hepatotoxic drugs showed sex differences in certain parameters in high-content imaging in a drug-specific fashion (60). In the case of APAP, toxicity was greater in pooled hepatocytes in suspension from postmenopausal women and was comparable to that seen in men, consistent with our findings. However, for other drugs, the female hepatocytes were more susceptible. Furthermore, the effects of sex on the metabolism of these drugs was not examined, and overall, the exposures were for very short time periods (up to 6 hours), making the results difficult to compare with those from in vivo studies. In addition, greater female representation in an APAP-induced acute liver failure (ALF) database has been reported, but this may be explained by a sex difference in the incidence of overdose $(61,62)$. However, evidence of a sex difference in human liver injury (female resistance) in ischemia/reperfusion injury and $\alpha 1$-antitrypsin deficiency has been reported (63-65). Thus, although the reciprocal relationship between $\mathrm{p} 53$ and $\mathrm{SAB}$ expression levels found in mouse liver is also present in humans, the contexts in which this could be relevant to human susceptibility to liver injury remain to be determined. However, since sustained JNK activation accompanies most acute and chronic liver disease, and since sustained JNK activation depends on the level of SAB expression, the SAB-dependent activation loop represents a potentially relevant therapeutic target to explore.

Lipoapoptosis may be one particularly translatable example. Lipotoxicity is also dependent on sustained JNK activation and SAB expression (13). We found resistance to palmitic acid toxicity in female versus male PMHs, which may be relevant to the progression of nonalcoholic steatohepatitis (NASH). However, future studies will be needed to determine whether the $\mathrm{ER} \alpha / \mathrm{p} 53 / \mathrm{mi}$ $R 34 a-5 p / S A B$ repression pathway contributes to the recently recognized resistance of premenopausal women to NASH $(6,66-70)$. Since premenopausal women exhibit decreased susceptibility to NASH and fibrosis, which is no longer the case in the postmenopausal stage, it is interesting to speculate that the postmenopausal increase in SAB levels may contribute to the pathogenesis of NASH. Furthermore, the postmenopausal increase in SAB levels further supports the role of estrogen in the repression of SAB. In addition, miR34a can be regulated by IRE-1 $\alpha$-dependent decay (RIDD) activation or inactivation, which may play a role in the expression of caspase 2 or SIRT1 (both targets of miR34a), depending on the context during the progression of NASH $(71,72)$.

Since miR34a-5p has other targets besides SAB, as noted above, it remains possible that their repression by $m i R 34 a-5 p$ also contributes to resistance to acute liver injury. SIRT1 in particular is a potentially relevant target, as it deacetylates and partially inhibits p53. A possible positive feedback effect of increased miR34a to increase p53 expression and to increase p53 activity (acetyl-p53) by repressing SIRT1 expression is plausible. Liver-specific Sirt1 knockout has been reported to protect against LPS-galactosamine toxicity (73). In addition, in PMHs, Sirt1 deletion, causing increased acetylation and activity of $\mathrm{p} 53$, protected against ActD/TNF-in- duced apoptosis. In contrast, Sirt1-transgenic mice were found to be protected from APAP toxicity (74), and induction of Sirt1 knockdown has been shown to worsen APAP toxicity (75). In addition, treatment with a SIRT1 activator has been shown to have no effect on early injury (6 hours) after APAP but decreases late injury (24 hours), while enhancing mitochondrial biogenesis and liver regeneration. Furthermore, resveratrol, which activates SIRT1, protected against APAP liver injury $(76,77)$. Taken together, conflicting evidence exists regarding the role of SIRT1 in acute injury models. However, we observed that basal SIRT1 levels were actually higher in female mouse and human livers and that $m i R 34 a^{\mathrm{i} \Delta \mathrm{Hep}}$ mice had no change in SIRT1 levels. This argues against an important role of SIRT1 repression in determining the sex difference in acute liver injury models. Therefore, the findings overall support the conclusion that repression of SAB expression by miR34a is the major determinant of resistance to liver injury in female mice.

One additional caveat is that GSH recovery is faster in APAP-treated female mice than in male mice. Therefore, the question is whether the faster GSH recovery dampens sustained JNK activation and protects, or whether decreased JNK activation is a consequence of less toxicity in females. We showed that there was no intrinsic sex difference in GSH recovery in response to nontoxic phorone-induced depletion. Furthermore, GCLC, the catalytic subunit of GCL, actually rapidly declined in APAP-treated male mice, possibly because of degradation, even before cell death begins in males, and correlated with sustained JNK activation. In contrast, GCLC protein did not increase or decrease in the female mice. Furthermore, in male mice treated with the miR34a-5p mimic, which decreases SAB protein levels, the recovery of GSH was accelerated, similar to what we observed in WT female mice. Conversely, miR34a deletion in females that expressed increased SAB expression led to an impaired GSH recovery comparable to that seen in WT male mice. Therefore, we conclude that the GSH recovery rate mainly reflects the severity of JNK activation and liver injury and is not the mechanism for sex difference in APAP toxicity.

In conclusion, the level of $\mathrm{SAB}$ expression in mitochondria plays a pivotal role in acute liver injury by mediating the extent of sustained activation of JNK. Evaluation of the effect of a graded increase in SAB expression in $S a b^{\mathrm{i} \triangle \mathrm{Hep}}$ mice has revealed a direct correlation between the level of SAB expression and the extent of liver injury. The interaction of JNK with $S A B$ is required for this effect of $\mathrm{SAB}$, and $\mathrm{SAB}$ is required to sustain this activation. Female mice expressed markedly lower levels of SAB, accounting for the resistance to acute injury in different liver injury models. SAB expression was low in females due to an ER $\alpha \rightarrow \uparrow \mathrm{p} 53$ $\rightarrow \uparrow m i R 34 a-5 p \rightarrow \downarrow \mathrm{SAB}$ pathway, in which the translation of $\mathrm{Sab}$ mRNA was presumably inhibited by targeting of the coding region by $m i R 34 a-5 p$ (Figure 7E). In support of the importance of the level of SAB expression as a determinant of liver injury, activation of $\mathrm{ER} \alpha$ in male mice increased p53 and miR34a-5p, which repressed $\mathrm{SAB}$ expression and protected from liver injury. Conversely, inhibition of ER $\alpha$ in female mice decreased p53 and miR34a-5p levels, which increased SAB expression, overcoming the female resistance to acute liver injury. In future studies, it will be of great interest to examine whether the sex difference seen in hepatic SAB is present in nonhepatic tissues and to identify the role of SAB in extrahepatic organ injury. 


\section{Methods}

Further information can be found in the Supplemental Methods, available online with this article.

Mice. Male and female C57BL/6NHsd mice (6-8 weeks of age) were purchased from Envigo and bred to obtain littermates. Ten- to twelve-week-old cage littermates were used for experiments. Jnk $1^{f / f l}$ and $J n k 2^{f / f l l}$ (C57BL/6J strain) mice were generated by Roger Davis (University of Massachusetts Medical School, Worcester, Massachusetts, USA), as described previously (6). Jnk1 $1^{f / f l}$ and $J n k 2^{f l / f l}$ mice were crossbred to obtain $J n k 1 / 2^{f / f l}$ littermates. Sab ${ }^{f / f l}$ mice (C57BL/6NHsd strain) were generated as described previously (11). Sa $b^{f l f l}$ mice

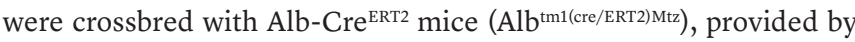
Pierre Chambon (Institut de Genetique et de Biologie Moleculaire et Cellulaire, Strasbourg, France) to generate tamoxifen-inducible hepatocyte-specific $S a b$-knockout mice ( $S a b^{\text {i४Hep}) ~(11) . ~ M i c e ~ w e r e ~}$ crossbred with $S a b^{f / f l}$ mice for more than 20 generations. miR34 $a^{\mathrm{f} /+}$ and $m i R 34 a^{+/+}$littermate mice were purchased from The Jackson laboratory and bred to obtain $m i R 34 a^{f l / f l}$ mice (C57BL/6J strain). Inducible hepatocyte-specific knockout $J n k 1 / 2^{\mathrm{i} \Delta \mathrm{Hep}}$ or $m i R 34 a^{\mathrm{i} \Delta \mathrm{Hep}}$ mice were obtained by tail-vein injection of AAV8-TBG-Cre (purchased from the University of Pennsylvania) into $J n k 1 / 2^{f / f l}$ and $m i R 34 a^{f / f l}$ mice, respectively. All experiments were performed using cage littermates. In all experiments, the substrain background of the mice was carefully controlled.

Human liver tissue biopsy samples. Human liver tissue biopsy samples were obtained from the Duke University Health Systems (DUHS) Nonalcoholic Fatty Liver Disease (NAFLD)Clinical Database, which was established in 2007 and is a prospective, open-enrollment, well-annotated clinical database of patients who underwent clinical and histological evaluations of the suspected diagnosis of NAFLD. Details of the DUHS NAFLD clinical database are described in a previous publication (78). The database is also linked to tissue repositories in which biosamples (e.g., blood, liver tissues) were collected at the time of the percutaneous liver biopsies or intraoperative needle liver biopsies in cases in which the patient underwent bariatric surgery.

Cases with normal or minimal nonspecific histologic changes were identified in the database. Individuals with a diagnosis of diabetes mellitus, as well as those who had undergone bariatric surgery or liver transplantation, were excluded from the case selection. Menopausal status was defined as a natural cessation of menstruation (no menstruation for more than 12 months) or a history of bilateral oophorectomy, using self-reported information collected via a standardized research questionnaire as a part of the DUHS NAFLD Clinical Database study. The menopause status was then confirmed by serum FSH. Cases involving women with a history of oral contraceptive use or hormone replacement therapy were excluded. After the exclusion, 5 premenopausal women, 3 postmenopausal women, and 8 age- and BMI-matched men were randomly selected. Age and BMI differences for female-male pairs were $2.6 \pm 1.9$ (range: 0-6 years) and $2.6 \pm 1.4 \mathrm{~km} / \mathrm{m}^{2}$ (range: $0.36-4.49 \mathrm{~km} / \mathrm{m}^{2}$ ). Statistical analysis of SAB expression of female-male pairs was performed using a paired, 2-tailed Student's $t$ test. A $P$ value of less than 0.05 was considered statistically significant.

$A A V$ and adenoviral vectors. Ad-lacZ and Ad-SAB were generated using the ViraPower Adenoviral Gateway Expression Kit (Invitrogen, Thermo Fisher Scientific) as described previously $(10,79)$. Adenoviral vectors with the CMV promotor were purified and concentrated using the Vivapure Adeno-PACK (Sartorius Stedim Biotech). Adenoviral titers were determined by infection of the viral vector into HEK293 cells (Invitrogen, Thermo Fisher Scientific) and staining of infected cells with adenoviral hexon antiserum (Santa Cruz Biotechnology). Different doses of adenoviral vectors were injected via the tail vein into male mice weighing $20 \mathrm{~g}$, and experiments were performed 10-14 days later. AAV8-TBG-GFP and AAV8-TBG-Cre were purchased from the Penn Vector Core/Addgene. Viral gene copies (GCs) $\left(\geq 2 \times 10^{12}\right)$ were injected via the tail vein into mice weighing $20 \mathrm{~g}$ or less, and experiments were performed 2 weeks later. For some experiments, AAV8-TBG-Cre and Ad-SAB $\left(0.5 \times 10^{9} \mathrm{IU}\right)$ were injected simultaneously. Ad-miR-GFP and the Ad-miR34a-5p mimic were purchased from Applied Biological Materials Inc. and amplified, purified, and concentrated in-house.

In vivo treatment with an $E R \alpha$ agonist and an $E R \alpha / \beta$ antagonist. Mice received the ER $\alpha$ agonist PPT ( $5 \mathrm{mg} / \mathrm{kg} /$ dose/day) in EtOH/corn oil subcutaneously 5 days per week for 2 weeks. In vivo experiments and PMH isolation were performed on the day following PPT treatment. Mice received the $\mathrm{ER} \alpha / \beta$ antagonist fulvestrant $(2$ or $5 \mathrm{mg} / \mathrm{kg}$ ) in EtOH/corn oil subcutaneously (1 dose per week for 3 weeks). Adenoviral vector injection was performed 2-3 days after the first dose of fulvestrant. PPT or fulvestrant was first dissolved in ethanol and diluted in corn oil (1:9 ratio), and the control animals received the vehicle as described previously $(80,81)$.

Small RNA sequence analysis. Liver tissue from 12-week-old chowfed C57BL/6N male and female littermates was snap-frozen in RNAlater RNA Stabilizing Solution (Thermo Fisher Scientific). miRNA was isolated using a QIAGEN miRNA Extraction Kit, and a miRNA library was generated using the QIAseq miRNA Library Kit (QIAGEN). Next-generation sequencing was performed using the Illumina 1 x75HT 30 GB. Mature miRNA-Seq analysis was performed on 3 independent libraries prepared from normal liver from 3 individual male and female C57BL/6N littermates. miRNA-Seq data were analyzed using Partek Flow, version 5 (Partek Inc.). Raw sequencing reads were first trimmed for adapters and then for the quality score (base positions with a Phred score $<20$ were trimmed from both ends, and trimmed reads shorter than $15 \mathrm{nt}$ were excluded from downstream analyses). Trimmed reads were mapped to the mouse genome mm10 using Bowtie (seed mismatch limit 1 and seed length 10). Aligned reads were then quantified to miRBase mature miRNAs v21 using the Partek E/M method. Finally, miRNAs with 5 raw read counts in at least 1 sample were normalized using upper-quartile normalization (82) with offset 1 and analyzed for differential expression using the Partek gene-specific analysis method. The differentially expressed miRNA lists (Gene Expression Omnibus [GEO] accession number GSE135299) were generated for each comparison using cutoffs of a FDR of less than 0.05 and fold changes of greater than 1.5 in either direction. The $P$ values were calculated on the basis of a Student's $t$ test of the replicate-normalized miRNA expression values for each miRNA in the female versus male groups. $P$ values of less than 0.05 indicate a significant change.

Statistics. A minimum of 3 biological replicates were analyzed for all studies. Data are expressed as the mean \pm SD or SEM. Statistical analyses were performed using the unpaired or paired, 2-tailed Student's $t$ test. Multivariate data were analyzed by 1-way ANOVA with Bonferroni's correction. A $P$ value of less than 0.05 was statistically significant. 
Study approval. Animal breeding and experiments were performed according to protocols approved by USC's IACUC and Institutional Biosafety Committee (IBC). All animals received care under the institutional guidelines. Human liver tissue biopsy and blood samples were used according to protocols approved by the IRBs of Duke University and USC. The DUHS NAFLD Clinical Database was approved by the Duke University IRB and established in accordance with Declaration of Helsinki ethical guidelines. All human subjects provided written informed consent prior to study enrollment.

\section{Author contributions}

SW, RWMM, CQC, JZ, FWMA, and TAT performed the experiments. YC, ML, SW, and TAT analyzed miRNA-Seq data. AS, MFA, YW, and AMD provided human liver samples. MA provided the ASO and GalNAc-ASO. RJD provided the $J n k 1^{f / f l}$ and $J n k 2^{f l / f l}$ mice. NK, TAT, and SW designed experiments and wrote the manuscript, and all authors read and provided input for the final version of the manuscript. SW and RWMM are co-first authors. SW appears first, as she assisted with obtaining institutional funding.

\section{Acknowledgments}

This research was supported by the NIH grants R01DK067215 (to NK) and RO1DK107220 (to RD); a Donald E. and Delia Baxter Foundation Faculty Fellows award (to SW); a USC Research Center for Liver Diseases Pilot Project award (to SW); and the USC Research Center for Liver Disease's Cell Separation and Culture, Cell and Tissue Imaging, Histology, and Metabolic, Analytical, and Instrumentation Cores (P30DK048522, to NK). The Duke NAFLD Clinical Database and Biorepository was supported by the Duke University Florence McAlister Professorship and the NAFLD Clinical Research Program. The bioinformatics software and computing resources used in the analysis were funded by the USC Office of Research and the Norris Medical Library of USC.

Address correspondence to: Neil Kaplowitz, Division of Gastroenterology and Liver Diseases, Department of Medicine, Keck School of Medicine, 2011 Zonal Avenue, HMR 101, Los Angeles, California 90033, USA. Phone: 323.442.5576; Email: kaplowit@usc.edu.
1. Gunawan BK, Liu ZX, Han D, Hanawa N, Gaarde WA, Kaplowitz N. c-Jun N-terminal kinase plays a major role in murine acetaminophen hepatotoxicity. Gastroenterology. 2006;131(1):165-178.

2. Hanawa N, Shinohara M, Saberi B, Gaarde WA, Han D, Kaplowitz N. Role of JNK translocation to mitochondria leading to inhibition of mitochondria bioenergetics in acetaminophen-induced liver injury. J Biol Chem. 2008;283(20):13565-13577.

3. Zhang J, et al. The role of MAP2 kinases and p38 kinase in acute murine liver injury models. Cell Death Dis. 2017;8(6):e2903.

4. Sharma M, Gadang V, Jaeschke A. Critical role for mixed-lineage kinase 3 in acetaminophen-induced hepatotoxicity. Mol Pharmacol. 2012;82(5):1001-1007.

5. Nakagawa H, et al. Deletion of apoptosis signal-regulating kinase 1 attenuates acetaminophen-induced liver injury by inhibiting c-Jun $\mathrm{N}$-terminal kinase activation. Gastroenterology. 2008;135(4):1311-1321.

6. Vernia S, et al. The PPAR $\alpha$-FGF21 hormone axis contributes to metabolic regulation by the hepatic JNK signaling pathway. Cell Metab. 2014;20(3):512-525.

7. Czaja MJ. JNK regulation of hepatic manifestations of the metabolic syndrome. Trends Endocrinol Metab. 2010;21(12):707-713.

8. Du K, Xie Y, McGill MR, Jaeschke H. Pathophysiological significance of c-jun $\mathrm{N}$-terminal kinase in acetaminophen hepatotoxicity. Expert Opin Drug Metab Toxicol. 2015;11(11):1769-1779.

9. Win S, Than TA, Zhang J, Oo C, Min RWM, Kaplowitz N. New insights into the role and mechanism of c-Jun-N-terminal kinase signaling in the pathobiology of liver diseases. Hepatology. 2018;67(5):2013-2024.

10. Win S, Than TA, Han D, Petrovic LM, Kaplowitz N. c-Jun N-terminal kinase (JNK)-dependent acute liver injury from acetaminophen or tumor necrosis factor (TNF) requires mitochondrial Sab protein expression in mice. J Biol Chem. 2011;286(40):35071-35078.

11. Win S, Than TA, Min RW, Aghajan M, Kaplowitz
N. c-Jun N-terminal kinase mediates mouse liver injury through a novel Sab (SH3BP5)-dependent pathway leading to inactivation of intramitochondrial Src. Hepatology. 2016;63(6):1987-2003.

12. Win S, Than TA, Fernandez-Checa JC, Kaplowitz $\mathrm{N}$. JNK interaction with Sab mediates ER stress induced inhibition of mitochondrial respiration and cell death. Cell Death Dis. 2014;5:e989.

13. Win S, Than TA, Le BH, García-Ruiz C, Fernandez-Checa JC, Kaplowitz N. Sab (Sh3bp5) dependence of JNK mediated inhibition of mitochondrial respiration in palmitic acid induced hepatocyte lipotoxicity. J Hepatol. 2015;62(6):1367-1374.

14. Chambers JW, LoGrasso PV. Mitochondrial c-Jun N-terminal kinase (JNK) signaling initiates physiological changes resulting in amplification of reactive oxygen species generation. J Biol Chem. 2011;286(18):16052-16062.

15. Dai G, He L, Chou N, Wan YJ. Acetaminophen metabolism does not contribute to gender difference in its hepatotoxicity in mouse. Toxicol Sci. 2006;92(1):33-41.

16. Rohrer PR, Rudraiah S, Goedken MJ, Manautou JE. Is nuclear factor erythroid 2-related factor 2 responsible for sex differences in susceptibility to acetaminophen-induced hepatotoxicity in mice? Drug Metab Dispos. 2014;42(10):1663-1674.

17. Du K, Williams CD, McGill MR, Jaeschke H. Lower susceptibility of female mice to acetaminophen hepatotoxicity: Role of mitochondrial glutathione, oxidant stress and c-jun N-terminal kinase. Toxicol Appl Pharmacol. 2014;281(1):58-66.

18. Kaplowitz N. Idiosyncratic drug hepatotoxicity. Nat Rev Drug Discov. 2005;4(6):489-499.

19. Saito C, Lemasters JJ, Jaeschke H. c-Jun N-terminal kinase modulates oxidant stress and peroxynitrite formation independent of inducible nitric oxide synthase in acetaminophen hepatotoxicity. Toxicol Appl Pharmacol.2010;246(1-2):8-17.

20. Jaeschke H, Gores GJ, Cederbaum AI, Hinson JA, Pessayre D, Lemasters JJ. Mechanisms of hepatotoxicity. Toxicol Sci. 2002;65(2):166-176.

21. Deng Y, Ren X, Yang L, Lin Y, Wu X. A JNK- dependent pathway is required for TNFalphainduced apoptosis. Cell. 2003;115(1):61-70.

22. Putcha GV, et al. JNK-mediated BIM phosphorylation potentiates BAX-dependent apoptosis. Neuron. 2003;38(6):899-914.

23. Fan M, Goodwin M, Vu T, Brantley-Finley C, Gaarde WA, Chambers TC. Vinblastine-induced phosphorylation of $\mathrm{Bcl}-2$ and $\mathrm{Bcl}-\mathrm{XL}$ is mediated by JNK and occurs in parallel with inactivation of the Raf-1/MEK/ERK cascade. J Biol Chem. 2000;275(39):29980-29985

24. Inoshita S, et al. Phosphorylation and inactivation of myeloid cell leukemia 1 by JNK in response to oxidative stress. J Biol Chem. 2002;277(46):43730-43734.

25. Chen D, et al. p53 Up-regulated Modulator of Apoptosis induction mediates acetaminophen-induced necrosis and liver injury in mice. Hepatology. 2019;69(5):2164-2179.

26. Dhanasekaran DN, Reddy EP. JNK-signaling: a multiplexing hub in programmed cell death. Genes Cancer. 2017;8(9-10):682-694.

27. Bourdi M, Davies JS, Pohl LR. Mispairing C57BL/6 substrains of genetically engineered mice and wild-type controls can lead to confounding results as it did in studies of JNK2 in acetaminophen and concanavalin A liver injury. Chem Res Toxicol. 2011;24(6):794-796.

28. Duan L, et al. Differential susceptibility to acetaminophen-induced liver injury in sub-strains of C57BL/6 mice: 6N versus 6J. Food Chem Toxicol. 2016;98(Pt B):107-118.

29. Shinohara $M$, et al. Silencing glycogen synthase kinase-3beta inhibits acetaminophen hepatotoxicity and attenuates JNK activation and loss of glutamate cysteine ligase and myeloid cell leukemia sequence 1. J Biol Chem. 2010;285(11):8244-8255.

30. Hewitt SC, Korach KS. Estrogen receptors: new directions in the new millennium. Endocr Rev. 2018;39(5):664-675.

31. Chow JD, Jones ME, Prelle K, Simpson ER, Boon WC. A selective estrogen receptor $\alpha$ agonist ameliorates hepatic steatosis in the male aromatase knockout mouse. J Endocrinol. 2011;210(3):323-334. 
32. Kuiper GG, et al. Comparison of the ligand binding specificity and transcript tissue distribution of estrogen receptors alpha and beta. Endocrinology. 1997;138(3):863-870.

33. Mårtensson UE, et al. Deletion of the G protein-coupled receptor 30 impairs glucose tolerance, reduces bone growth, increases blood pressure, and eliminates estradiol-stimulated insulin release in female mice. Endocrinology. 2009;150(2):687-698.

34. Osborne CK, Wakeling A, Nicholson RI. Fulvestrant: an oestrogen receptor antagonist with a novel mechanism of action. Br JCancer. 2004; 90 Suppl 1:S2-S6.

35. Lai AC, Crews CM. Induced protein degradation: an emerging drug discovery paradigm. Nat Rev Drug Discov. 2017;16(2):101-114.

36. Kraichely DM, Sun J, Katzenellenbogen JA, Katzenellenbogen BS. Conformational changes and coactivator recruitment by novel ligands for estrogen receptor-alpha and estrogen receptor-beta: correlations with biological character and distinct differences among SRC coactivator family members. Endocrinology. 2000;141(10):3534-3545.

37. Petrie WK, et al. G protein-coupled estrogen receptor-selective ligands modulate endometrial tumor growth. Obstet Gynecol Int. 2013;2013:472720.

38. Berger C, Qian Y, Chen X. The p53-estrogen receptor loop in cancer. Curr Mol Med. 2013;13(8):1229-1240

39. Ito A, et al. p300/CBP-mediated p 53 acetylation is commonly induced by p53-activating agents and inhibited by MDM2. EMBO J. 2001;20(6):1331-1340.

40. Lee TK, Lau TC, Ng IO. Doxorubicin-induced apoptosis and chemosensitivity in hepatoma cell lines. Cancer Chemother Pharmacol. 2002;49(1):78-86.

41. Seitz SJ, et al. Chemotherapy-induced apoptosis in hepatocellular carcinoma involves the p53 family and is mediated via the extrinsic and the intrinsic pathway. Int J Cancer. 2010;126(9):2049-2066.

42. Yamakuchi M, Ferlito M, Lowenstein CJ. miR-34a repression of SIRT1 regulates apoptosis. Proc Natl Acad Sci USA. 2008;105(36):13421-13426.

43. Vaziri $\mathrm{H}$, et al. hSIR2(SIRT1) functions as an NAD-dependent p53 deacetylase. Cell. 2001;107(2):149-159.

44. Zhao Y, et al. Acetylation of p53 at lysine 373/382 by the histone deacetylase inhibitor depsipeptide induces expression of $\mathrm{p} 21$ (Waf1/Cip1). Mol Cell Biol. 2006;26(7):2782-2790.

45. Huo Y, et al. Protective role of p53 in acetaminophen hepatotoxicity. Free Radic Biol Med. 2017;106:111-117.

46. Navarro F, Lieberman J. miR-34 and p53: new insights into a complex functional relationship. PLOS ONE. 2015;10(7):e0132767.

47. Graham MJ, et al. Cardiovascular and metabolic effects of ANGPTL3 antisense oligonucleotides. N Engl JMed. 2017;377(3):222-232.

48. Cazanave SC, et al. JNK1-dependent PUMA expression contributes to hepatocyte lipoapoptosis. J Biol Chem. 2009;284(39):26591-26602.

49. Wiltshire C, Matsushita M, Tsukada S, Gillespie DA,
May GH. A new c-Jun N-terminal kinase (JNK)interacting protein, $\mathrm{Sab}$ (SH3BP5), associates with mitochondria. Biochem J. 2002;367(Pt 3):577-585.

50. Cubero FJ, et al. Combined activities of JNK1 and JNK2 in hepatocytes protect against toxic liver injury. Gastroenterology. 2016;150(4):968-981.

51. Zoubek ME, et al. Protective role of c-Jun N-terminal kinase-2 (JNK2) in ibuprofen-induced acute liver injury. J Pathol. 2019;247(1):110-122.

52. Chambers TP, Santiesteban L, Gomez D, Chambers JW. Sab mediates mitochondrial dysfunction involved in imatinib mesylate-induced cardiotoxicity. Toxicology. 2017;382:24-35.

53. Chambers TP, Portalatin GM, Paudel I, Robbins CJ, Chambers JW. Sub-chronic administration of LY294002 sensitizes cervical cancer cells to chemotherapy by enhancing mitochondrial JNK signaling. Biochem Biophys Res Commun. 2015;463(4):538-544.

54. Paudel I, Hernandez SM, Portalatin GM, Chambers TP, Chambers JW. Sab concentrations indicate chemotherapeutic susceptibility in ovarian cancer cell lines. Biochem J. 2018;475(21):3471-3492.

55. Chang TC, et al. Transactivation of miR-34a by p53 broadly influences gene expression and promotes apoptosis. Mol Cell. 2007;26(5):745-752.

56. Li XJ, Ren ZJ, Tang JH. MicroRNA-34a: a potential therapeutic target in human cancer. Cell Death Dis. 2014;5:e1327.

57. Fuchs SY, Adler V, Pincus MR, Ronai Z. MEKK1/ JNK signaling stabilizes and activates p53. Proc Natl Acad Sci USA . 1998;95(18):10541-10546.

58. Shi Y, et al. ROS-dependent activation of JNK converts $\mathrm{p} 53$ into an efficient inhibitor of oncogenes leading to robust apoptosis. Cell Death Differ. 2014;21(4):612-623.

59. Saha MN, et al. Targeting p53 via JNK pathway: a novel role of RITA for apoptotic signaling in multiple myeloma. PLoS ONE. 2012;7(1):e30215.

60. Mennecozzi M, Landesmann B, Palosaari T, Harris G, Whelan M. Sex differences in liver toxicity-do female and male human primary hepatocytes react differently to toxicants in vitro? PLoS ONE. 2015;10(4):e0122786.

61. Fontana RJ. Acute liver failure including acetaminophen overdose. Med Clin North Am. 2008;92(4):761-794, viii.

62. Rubin JB, Hameed B, Gottfried M, Lee WM, Sarkar M. Acute Liver Failure Study Group. Acetaminophen-induced acute liver failure is more common and more severe in women. Clin Gastroenterol Hepatol. 2018;16(6):936-946.

63. McCully JD, Toyoda Y, Wakiyama H, Rousou AJ, Parker RA, Levitsky S. Age- and gender-related differences in ischemia/reperfusion injury and cardioprotection: effects of diazoxide. Ann Thorac Surg. 2006;82(1):117-123.

64. Murphy E, Steenbergen C. Gender-based differences in mechanisms of protection in myocardial ischemia-reperfusion injury. Cardiovasc Res. 2007;75(3):478-486.

65. Chu AS, Chopra KB, Perlmutter DH. Is severe progressive liver disease caused by alpha-1-antitrypsin deficiency more common in children or adults? Liver Transpl. 2016;22(7):886-894.

66. Suzuki A. NASH in special populations. Gastroenterol Hepatol (N Y). 2014;10(4):262-264.

67. Yang JD, et al. Gender and menopause impact severity of fibrosis among patients with nonalcoholic steatohepatitis. Hepatology. 2014;59(4):1406-1414.

68. Klair JS, et al. A longer duration of estrogen deficiency increases fibrosis risk among postmenopausal women with nonalcoholic fatty liver disease. Hepatology. 2016;64(1):85-91.

69. Ballestri S, Nascimbeni F, Baldelli E, Marrazzo A, Romagnoli D, Lonardo A. NAFLD as a sexual dimorphic disease: role of gender and reproductive status in the development and progression of nonalcoholic fatty liver disease and inherent cardiovascular risk. Adv Ther. 2017;34(6):1291-1326.

70. Lonardo A, et al. Sex differences in nonalcoholic fatty liver disease: state of the art and identification of research gaps [published online ahead of print March 29, 2019]. Hepatology. doi: 10.1002/hep.30626.

71. Upton JP, et al. IRE1 $\alpha$ cleaves select microRNAs during ER stress to derepress translation of proapoptotic Caspase-2. Science. 2012;338(6108):818-822.

72. Yamakuchi M. MicroRNA regulation of SIRT1. Front Physiol. 2012;3:68.

73. Cui $\mathrm{X}$, et al. Inactivation of Sirt1 in mouse livers protects against endotoxemic liver injury by acetylating and activating NF-кB. Cell Death Dis. 2016;7(10):e2403.

74. Rada P, et al. SIRT1 controls acetaminophen hepatotoxicity by modulating inflammation and oxidative stress. Antioxid Redox Signal. 2018;28(13):1187-1208.

75. Liu X, et al. Acetaminophen responsive miR19b modulates SIRT1/Nrf2 signaling pathway in drug-induced hepatotoxicity. Toxicol Sci. 2019;170(2):476-488.

76. Wang Y, et al. Hepato-protective effect of resveratrol against acetaminophen-induced liver injury is associated with inhibition of CYP-mediated bioactivation and regulation of SIRT1-p53 signaling pathways. Toxicol Lett. 2015;236(2):82-89.

77. Wojnarová L, Kutinová Canová N, Farghali H, Kučera T. Sirtuin 1 modulation in rat model of acetaminophen-induced hepatotoxicity. Physiol Res. 2015;64 Suppl 4:S477-S487.

78. Youssef NA, et al. Associations of depression, anxiety and antidepressants with histological severity of nonalcoholic fatty liver disease. Liver Int. 2013;33(7):1062-1070.

79. Than TA, Lou H, Ji C, Win S, Kaplowitz N. Role of cAMP-responsive element-binding protein (CREB)-regulated transcription coactivator 3 (CRTC3) in the initiation of mitochondrial biogenesis and stress response in liver cells. J Biol Chem. 2011;286(25):22047-22054.

80. Miller NR, Jover T, Cohen HW, Zukin RS, Etgen AM. Estrogen can act via estrogen receptor alpha and beta to protect hippocampal neurons against global ischemia-induced cell death. Endocrinology. 2005;146(7):3070-3079.

81. Ariazi EA, et al. Emerging principles for the development of resistance to antihormonal therapy: implications for the clinical utility of fulvestrant. JSteroid Biochem Mol Biol. 2006;102(1-5):128-138.

82. Bullard JH, Purdom E, Hansen KD, Dudoit S. Evaluation of statistical methods for normalization and differential expression in mRNA-Seq experiments. BMC Bioinformatics. 2010;11:94. 